TRANSACTIONS OF THE

AMERICAN MATHEMATICAL SOCIETY

Volume 354, Number 9, Pages 3409-3433

S 0002-9947(02)03021-0

Article electronically published on May 8, 2002

\title{
LOWER CENTRAL SERIES AND FREE RESOLUTIONS OF HYPERPLANE ARRANGEMENTS
}

\author{
HENRY K. SCHENCK AND ALEXANDER I. SUCIU
}

\begin{abstract}
If $M$ is the complement of a hyperplane arrangement, and $A=$ $H^{*}(M, \mathbb{k})$ is the cohomology ring of $M$ over a field $\mathbb{k}$ of characteristic 0 , then the ranks, $\phi_{k}$, of the lower central series quotients of $\pi_{1}(M)$ can be computed from

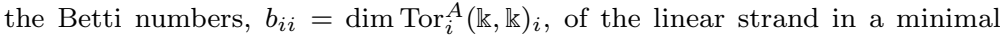
free resolution of $\mathbb{k}$ over $A$. We use the Cartan-Eilenberg change of rings spectral sequence to relate these numbers to the graded Betti numbers, $b_{i j}^{\prime}=$ $\operatorname{dim} \operatorname{Tor}_{i}^{E}(A, \mathbb{k})_{j}$, of a minimal resolution of $A$ over the exterior algebra $E$.

From this analysis, we recover a formula of Falk for $\phi_{3}$, and obtain a new formula for $\phi_{4}$. The exact sequence of low-degree terms in the spectral sequence allows us to answer a question of Falk on graphic arrangements, and also shows that for these arrangements, the algebra $A$ is Koszul if and only if the arrangement is supersolvable.

We also give combinatorial lower bounds on the Betti numbers, $b_{i, i+1}^{\prime}$, of the linear strand of the free resolution of $A$ over $E$; if the lower bound is attained for $i=2$, then it is attained for all $i \geq 2$. For such arrangements, we compute the entire linear strand of the resolution, and we prove that all components of the first resonance variety of $A$ are local. For graphic arrangements (which do not attain the lower bound, unless they have no braid subarrangements), we show that $b_{i, i+1}^{\prime}$ is determined by the number of triangles and $K_{4}$ subgraphs in the graph.
\end{abstract}

\section{INTRODUCTION}

1.1. LCS formulas. The lower central series (LCS) of a finitely-generated group $G$ is a chain of normal subgroups, $G=G_{1} \geq G_{2} \geq G_{3} \geq \cdots$, defined inductively by $G_{k}=\left[G_{k-1}, G\right]$. Fix a field $\mathbb{k}$ of characteristic 0 . Associated to $G$ is a graded Lie algebra, $\operatorname{gr}(G) \otimes \mathbb{k}:=\bigoplus_{k=1}^{\infty} G_{k} / G_{k+1} \otimes \mathbb{k}$, with Lie bracket induced by the commutator map. Let $\phi_{k}=\phi_{k}(G)$ denote the rank of the $k$-th quotient.

One case in which the numbers $\phi_{k}$ have aroused great interest occurs when $M$ is the complement of a complex hyperplane arrangement and $G=\pi_{1}(M)$ is its fundamental group. In certain situations, the celebrated lower central series formula holds:

$$
\prod_{k=1}^{\infty}\left(1-t^{k}\right)^{\phi_{k}}=P(M,-t)
$$

Received by the editors August 22, 2001 and, in revised form, September 19, 2001.

2000 Mathematics Subject Classification. Primary 16E05, 20F14, 52C35; Secondary 16S37.

Key words and phrases. Lower central series, free resolution, hyperplane arrangement, change of rings spectral sequence, Koszul algebra, linear strand, graphic arrangement.

The first author was partially supported by an NSF postdoctoral research fellowship.

The second author was partially supported by NSF grant DMS-0105342. 
where $P(M, t)=\sum b_{i} t^{i}$ is the Poincaré polynomial of the complement. This formula was first proved by Kohno [21] for braid arrangements, and then generalized by Falk and Randell 15] to supersolvable arrangements. In [29, Shelton and Yuzvinsky gave a beautiful interpretation of the LCS formula in terms of Koszul duality. The LCS formula holds for rational $K(\pi, 1)$ spaces (see Papadima and Yuzvinsky [26]), and an analogue holds for hypersolvable arrangements (see Jambu and Papadima [20]).

All these LCS formulas ultimately hinge on the assumption that the cohomology algebra (or its quadratic closure) is Koszul. The goal of this paper is to explore formulas of type (1.1) for arrangements where such assumptions fail. In complete generality, there is no good substitute for the polynomial $P(X,-t)$ on the right side, as illustrated by the non-Fano plane (see Peeva [27]). But, as conjectured in 31], and as verified here in certain situations (at least in low degrees), there do exist large classes of arrangements for which a modified LCS formula holds, with the Poincaré polynomial replaced by another (combinatorially determined) polynomial.

1.2. LCS ranks and Betti numbers of the linear strand. Our starting point is a formula expressing the LCS ranks of an arrangement group in terms of the Betti numbers, $b_{i i}=\operatorname{dim}_{\mathbb{k}} \operatorname{Ext}_{A}^{i}(\mathbb{k}, \mathbb{k})_{i}=\operatorname{dim}_{\mathbb{k}} \operatorname{Tor}_{i}^{A}(\mathbb{k}, \mathbb{k})_{i}$, of the linear strand of the free resolution of the residue field $\mathbb{k}$, viewed as a module over the Orlik-Solomon algebra, $A=H^{*}(M, \mathbb{k})$ :

$$
\prod_{k=1}^{\infty}\left(1-t^{k}\right)^{-\phi_{k}}=\sum_{i=0}^{\infty} b_{i i} t^{i}
$$

A bit of history: In [21], Kohno showed that the left-hand quantity is equal to the Hilbert series of the universal enveloping algebra, $U=U(\mathfrak{g})$, of the holonomy Lie algebra $\mathfrak{g}$ of the complement $M$. (This follows from the Poincaré-Birkhoff-Witt theorem, together with the isomorphism $\operatorname{gr}(G) \otimes \mathbb{k} \cong \mathfrak{g}$, which is a consequence of the formality of $M$, in the sense of Sullivan.) In [29], Shelton and Yuzvinsky proved that $U=\bar{A}^{!}$, the Koszul dual of the quadratic closure of the Orlik-Solomon algebra. Finally, results of Priddy [28] and Löfwall [22] relate the Koszul dual of a quadratic algebra to the linear strand in the Yoneda Ext-algebra:

$$
\bar{A} \cong \bigoplus_{i} \operatorname{Ext} \frac{i}{A}(\mathbb{k}, \mathbb{k})_{i}
$$

Since obviously $\operatorname{Ext} \frac{i}{A}(\mathbb{k}, \mathbb{k})_{i}=\operatorname{Ext}_{A}^{i}(\mathbb{k}, \mathbb{k})_{i}$, formula (1.2) follows at once (compare [27, Theorem 2.6]).

If $A$ is a Koszul algebra (i.e., $\operatorname{Ext}_{A}^{i}(\mathbb{k}, \mathbb{k})_{j}=0$, for $i \neq j$ ), then $A=\bar{A}$, and the Koszul duality formula, $\operatorname{Hilb}\left(A^{!}, t\right) \cdot \operatorname{Hilb}(A,-t)=1$, yields the LCS formula (1.1). This computation applies to supersolvable arrangements, which do have Koszul OS-algebras, cf. [29]. The more general formula (1.2) was first exploited by Peeva in 27] to obtain bounds on the LCS ranks $\phi_{k}$.

1.3. The Orlik-Solomon algebra and free resolutions. A minimal free resolution of a graded module $N$ over a graded $\mathbb{k}$-algebra $R$ is simply a graded exact sequence

$$
P_{\bullet}: \quad \cdots \longrightarrow \bigoplus_{j} R^{b_{2 j}}(-j) \longrightarrow \bigoplus_{j} R^{b_{1 j}}(-j) \longrightarrow \bigoplus_{j} R^{b_{0 j}}(-j) \longrightarrow N \rightarrow 0
$$


where the exponents are $b_{i j}(N)=\operatorname{dim}_{\mathbb{k}} \operatorname{Tor}_{i}^{R}(N, \mathbb{k})_{j}$. We are especially interested in two cases:

- $N=A=E / I$, the Orlik-Solomon algebra of an arrangement of $n$ hyperplanes, and $R=E$ is the exterior algebra on $n$ generators, in which case we will write $b_{i j}^{\prime}=\operatorname{dim}_{\mathbb{k}} \operatorname{Tor}_{i}^{E}(A, \mathbb{k})_{j}$.

- $N=\mathbb{k}$ and $R=A$, in which case we will write $b_{i j}=\operatorname{dim}_{\mathbb{k}} \operatorname{Tor}_{i}^{A}(\mathbb{k}, \mathbb{k})_{j}$.

We study the relationship between these two free resolutions by means of the change of rings spectral sequence associated to the composition $E \rightarrow A \rightarrow \mathbb{k}$ :

$$
\operatorname{Tor}_{i}^{A}\left(\operatorname{Tor}_{j}^{E}(A, \mathbb{k}), \mathbb{k}\right) \Longrightarrow \operatorname{Tor}_{i+j}^{E}(\mathbb{k}, \mathbb{k}) .
$$

The goal is to translate the right-hand side of formula (1.2) into an expression involving the resolution of $A$ over $E$, which is a much smaller (in the sense that the ranks of the free modules appearing in the resolution are much smaller) object than the resolution of $\mathbb{k}$ over $A$.

A resolution $P_{\bullet}$ as in (1.4) is linear if each free module $F_{i}$ is generated in a single degree $\alpha_{i}$ and $\alpha_{i}=\alpha_{i-1}-1$. By convention, when $N=R / J$ and $J$ is generated in degree $\geq 2$, the linear strand is the complex with terms $F_{i}=R^{b_{i, i+1}}(-i-1)$, $i \geq 1$. Most of the results we obtain from the change of rings spectral sequence stem from the happy fact that the resolution of $\mathbb{k}$ as a module over $E$ is linear (with $\left.\operatorname{dim}_{\mathbb{k}} \operatorname{Tor}_{i}^{E}(\mathbb{k}, \mathbb{k})_{i}=\left(\begin{array}{c}n+i-1 \\ i\end{array}\right)\right)$. In [10], Eisenbud, Popescu and Yuzvinsky prove that $A^{\vee}=H_{*}(M, \mathbb{k})$, viewed as an $E$-module via the cap product, has a linear free resolution; we had hoped that this would make one of the change of rings spectral sequences particularly simple, but it does not.

1.4. LCS ranks of arrangement groups. Let $a_{i}$ denote the number of minimal generators of degree $i$ in the Orlik-Solomon ideal $I$. It is elementary to see that $\phi_{1}=b_{1}$ and $\phi_{2}=a_{2}$. In [11, Falk gave a formula for $\phi_{3}$ of an arbitrary arrangement, which can be interpreted as $\phi_{3}=b_{23}^{\prime}$, but no general formula for $\phi_{4}$ was known (besides the one implicit in (1.2)). Using the above spectral sequence, we obtain a formula for $\phi_{4}$ which depends solely on the resolution of $A$ over $E$ :

$$
\phi_{4}=\left(\begin{array}{c}
a_{2} \\
2
\end{array}\right)+b_{34}^{\prime}-\delta_{4},
$$

where $\delta_{4}$ denotes the number of minimal quadratic syzygies on the degree 2 generators of $I$ which are Koszul syzygies. For many arrangements, this expression yields an explicit combinatorial formula for $\phi_{4}$.

1.5. Minimal linear strand arrangements. For arrangements with

$$
b_{23}^{\prime}=2 \sum_{X \in L_{2}(\mathcal{A})}\left(\begin{array}{c}
\mu(X)+1 \\
3
\end{array}\right),
$$

we have

$$
\phi_{4}=\sum_{X \in L_{2}(\mathcal{A})} \frac{\mu(X)^{2}\left(\mu(X)^{2}-1\right)}{4} .
$$

Arrangements for which (1.7) holds have $b_{i, i+1}^{\prime}$ as small as possible, for all $i \geq 2$ (given the combinatorics); in other words, the linear strand in the free resolution of $A$ over $E$ is minimal. In view of this, we call such arrangements minimal linear strand $(M L S)$ arrangements. Examples include Kohno's arrangements $X_{2}$ and $X_{3}$, the non-Pappus arrangement, and graphic arrangements with no $K_{4}$ subgraphs. 
For MLS arrangements in $\mathbb{C}^{3}$, we compute the whole Betti diagram of the minimal free resolution of $A$ over $E$, as well as the differentials in the linear strand.

As a generalization of formula (1.8), we conjecture that, for all MLS arrangements and all $k \geq 2$,

$$
\phi_{k}=\frac{1}{k} \sum_{d \mid k} \sum_{X \in L_{2}(\mathcal{A})} \mu(d) \mu(X)^{\frac{k}{d}},
$$

where $\mu$ stands for both the Möbius function of the integers, and the Möbius function of the intersection lattice of the arrangement. Equivalently,

$$
\prod_{k=1}^{\infty}\left(1-t^{k}\right)^{\phi_{k}}=(1-t)^{b_{1}-b_{2}} \prod_{X \in L_{2}(\mathcal{A})}(1-\mu(X) t) .
$$

The proof of the conjecture hinges upon computing the image of a $d_{2}$ map in the change of rings spectral sequence.

1.6. Graphic arrangements. As another application our methods, we study the free resolutions and LCS quotients of a graphic arrangement. Let $\mathrm{G}$ be a (simple) graph on $\ell$ vertices, with edge-set $\mathrm{E}$, and let $\mathcal{A}_{\mathrm{G}}=\left\{z_{i}-z_{j}=0 \mid(i, j) \in \mathrm{E}\right\}$ be the corresponding arrangement in $\mathbb{C}^{\ell}$. Using a well-known result of Stanley, we show that the OS-algebra $A$ is Koszul if and only if the arrangement $\mathcal{A}_{\mathrm{G}}$ is supersolvable.

As for the LCS ranks of the fundamental group of the complement of $\mathcal{A}_{\mathrm{G}}$, we conjecture that they are given by

$$
\phi_{k}=\frac{1}{k} \sum_{d \mid k} \sum_{j=1}^{k} \sum_{s=j}^{k}(-1)^{s-j}\left(\begin{array}{l}
s \\
j
\end{array}\right) \kappa_{s} \mu(d) j^{\frac{k}{d}},
$$

where $\kappa_{s}$ is the number of complete subgraphs on $s+1$ vertices; equivalently,

$$
\prod_{k=1}^{\infty}\left(1-t^{k}\right)^{\phi_{k}}=\prod_{j=1}^{\ell-1}(1-j t)^{\sum_{s=j}^{\ell-1}(-1)^{s-j}\left(\begin{array}{l}
s \\
j
\end{array}\right) \kappa_{s}}
$$

We verify this conjecture for $k \leq 3$. In particular, we find $\phi_{3}=2\left(\kappa_{2}+\kappa_{3}\right)$, thereby answering a question of Falk [14]. For $k=4$, we verify the inequality $\phi_{4} \geq 3\left(\kappa_{2}+3 \kappa_{3}+2 \kappa_{4}\right)$, and show that equality holds if $\kappa_{3}=0$.

Acknowledgment. The computations for this work were done primarily with the algebraic geometry and commutative algebra system Macaulay 2, by Grayson and Stillman [19]. Additional computations were done with Mathematica 4.0, from Wolfram Research.

\section{Resolution of the OS-Algebra over the EXterior Algebra}

We start by analyzing the minimal free resolution of the Orlik-Solomon algebra over the exterior algebra. For "minimal linear strand" arrangements, the graded Betti numbers of this resolution can be computed explicitly from the Möbius function of the intersection lattice. 
2.1. Orlik-Solomon algebra. Let $\mathcal{A}$ be a (central) arrangement of complex hyperplanes in $\mathbb{C}^{\ell}$, with complement $M(\mathcal{A})=\mathbb{C}^{\ell} \backslash \bigcup_{H \in \mathcal{A}} H$. Let $L(\mathcal{A})=\left\{\bigcap_{H \in \mathcal{B}} H \mid\right.$ $\mathcal{B} \subseteq \mathcal{A}\}$ be the intersection lattice of all flats of the arrangement, with order given by reverse inclusion, and rank given by codimension. The cohomology ring of $M(\mathcal{A})$ with coefficients in a field $\mathbb{k}$ is isomorphic to a certain $\mathbb{k}$-algebra, $A=A(\mathcal{A})$, which can be described solely in terms of $L(\mathcal{A})$, as follows (see the book by Orlik and Terao [25] as a general reference).

Let $E$ be the exterior algebra on generators $e_{1}, \ldots, e_{n}$ in degree 1, corresponding to the hyperplanes. This is a differential graded algebra over $\mathbb{k}$, with grading

$$
E_{r}=\operatorname{span}_{\mathbb{k}}\left\{e_{J}=e_{i_{1}} \wedge \cdots \wedge e_{i_{r}} \mid J=\left\{i_{1}, \ldots, i_{r}\right\} \subseteq\{1, \ldots, n\}\right\},
$$

and differential $\partial: E_{i} \rightarrow E_{i-1}$ given by $\partial(1)=0, \partial\left(e_{i}\right)=1$, together with the graded Leibnitz rule. The Orlik-Solomon algebra, $A$, is the quotient of $E$ by the (homogeneous) ideal

$$
I=\left(\partial e_{\mathcal{B}}\left|\operatorname{codim} \bigcap_{H \in \mathcal{B}} H<\right| \mathcal{B} \mid\right) .
$$

The algebra $A=E / I$ inherits a grading from $E$ in the obvious fashion. We denote the Betti numbers of the arrangement by $b_{i}=\operatorname{dim} A_{i}$. As is well known,

$$
b_{i}=\sum_{X \in L_{i}(\mathcal{A})}(-1)^{i} \mu(X),
$$

where $\mu: L(\mathcal{A}) \rightarrow \mathbb{Z}$ is the Möbius function of the lattice.

Let $a_{j}$ be the number of minimal generators of $I$ having degree exactly $j$, and let $I[j] \subseteq I$ be the ideal of $E$ generated by those $a_{j}$ elements. We have

$$
a_{j}=\operatorname{dim}_{\mathbb{k}}(I[j])_{j}=\operatorname{dim}_{\mathbb{k}} \operatorname{Tor}_{1}^{E}(A, \mathbb{k})_{j} .
$$

From the exact sequence $0 \rightarrow I \rightarrow E \rightarrow A \rightarrow 0$, we get $a_{j}+b_{j} \leq\left(\begin{array}{l}n \\ j\end{array}\right)$. Furthermore, since $I$ is generated in degree $\geq 2$, we have $a_{1}=0$ and

$$
a_{2}=\left(\begin{array}{l}
n \\
2
\end{array}\right)-b_{2}=\sum_{X \in L_{2}(\mathcal{A})}\left(\begin{array}{c}
\mu(X) \\
2
\end{array}\right) .
$$

If $A$ is quadratic (i.e., $I=I[2]$ ), then $a_{j}=0$ for $j>2$. No explicit combinatorial formula for the numbers $a_{j}(j>2)$ is known in general.

2.2. Free resolution of the OS-algebra. By general results on modules over the exterior algebra, the Orlik-Solomon algebra $A$ admits a resolution over $E$ by finitely-generated free modules,

$$
\cdots \longrightarrow F_{2} \longrightarrow F_{1} \longrightarrow E \longrightarrow A \longrightarrow 0 .
$$

Furthermore, there exist minimal free resolutions, in the sense that $\operatorname{dim}_{\mathbb{k}} F_{i}=$ $\operatorname{dim}_{\mathbb{k}} \operatorname{Tor}_{i}^{E}(A, \mathbb{k})$, for all $i \geq 1$. Such resolutions are unique up to chain-equivalence. Our goal in this section is to obtain a better understanding of the graded Betti numbers

$$
b_{i j}^{\prime}=\operatorname{dim}_{\mathbb{k}} \operatorname{Tor}_{i}^{E}(A, \mathbb{k})_{j} .
$$

We start with some simple observations. 
Lemma 2.3. Let $\mathcal{A}$ be an essential, central arrangement of rank $\ell$. If $j \geq i+\ell$, then

$$
\operatorname{Tor}_{i}^{E}(A, \mathbb{k})_{j}=0 .
$$

Proof. As in the proof of Theorem 1.1 of [10, to study the free $E$-resolution of $A$, we may pass to a decone of $\mathcal{A}$. Let $A^{\prime}=E^{\prime} / I^{\prime}$ be the OS-algebra of the decone. Then $A_{j}^{\prime}=0$, for $j \geq \ell$. The free $E^{\prime}$-resolution of $\mathbb{k}$ is linear, so after tensoring this resolution with $A^{\prime}$, we obtain a complex of modules with $i$-th term $\bigoplus A^{\prime}(-i)$, which vanishes in degrees $i+\ell$ and higher.

Lemma 2.4. Let $\mathcal{A}$ be an arrangement of n hyperplanes in $\mathbb{C}^{\ell}$. The Betti numbers of the minimal free resolution of $A$ over $E$ are related to the Betti numbers of $A$, as follows:

$$
\left(\sum_{i=0}^{\infty} \sum_{j=0}^{\ell-1}(-1)^{i} b_{i, i+j}^{\prime} t^{i+j}\right) \cdot(1+t)^{n}=\sum_{i=0}^{\ell} b_{i} t^{i}
$$

Proof. From (2.4), we get $\left(\sum_{i}(-1)^{i} \operatorname{Hilb}\left(F_{i}, t\right)\right) \cdot P(E, t)=P(A, t)$.

2.5. Local syzygies. We now analyze in more detail a certain type of linear syzygy in the resolution of $A$ over $E$. These "local" syzygies come from flats in $L_{2}^{\prime}(\mathcal{A}):=$ $\left\{X \in L_{2}(\mathcal{A}) \mid \mu(X)>1\right\}$. We start with the simplest situation.

Example 2.6 (Pencil of 3 lines). Let $\mathcal{A}=\left\{H_{0}, H_{1}, H_{2}\right\}$ be an arrangement of 3 lines through the origin of $\mathbb{C}^{2}$. The Orlik-Solomon ideal is generated by $\partial e_{012}=$ $\left(e_{1}-e_{2}\right) \wedge\left(e_{0}-e_{2}\right)$. It is readily seen that the minimal free resolution of $A$ over $E$ is

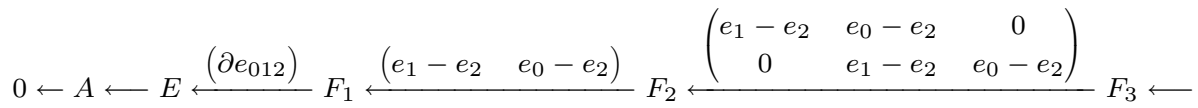

$$
\begin{aligned}
& \cdots \longleftarrow F_{i} \stackrel{\left(\begin{array}{cccccc}
e_{1}-e_{2} & e_{0}-e_{2} & 0 & \cdots & 0 & 0 \\
0 & e_{1}-e_{2} \ldots e_{0}-e_{2} \ldots \ldots & 0 & 0 \\
0 & 0 & 0 & \cdots \cdots \cdots \cdots \cdots \cdots \cdots \cdots & e_{1}-e_{2} & e_{0}-e_{2}
\end{array}\right)}{F_{i+1} \longleftarrow \cdots}
\end{aligned}
$$

with $F_{i}=E^{i}(-i)$. Thus, $b_{i, i+1}^{\prime}=i$ for $i \geq 1$, and $b_{i, i+r}^{\prime}=0$ for $r>1$.

Example 2.7 (Pencils of $n+1$ hyperplanes). More generally, let $\mathcal{A}=\left\{H_{0}, \ldots\right.$, $\left.H_{n}\right\}$ be a central arrangement of $n+1 \geq 3$ hyperplanes whose common intersection is of codimension two. The Orlik-Solomon ideal is generated by all elements $\partial e_{i j k}=$ $\left(e_{j}-e_{i}\right) \wedge\left(e_{k}-e_{i}\right)$ with $0 \leq i<j<k \leq n$. In fact, $I=I[2]$, with minimal set of generators $\left(e_{j}-e_{0}\right) \wedge\left(e_{k}-e_{0}\right)$, for $1 \leq j<k \leq n$.

The minimal resolution of $A$ over $E$ has a form similar to the one above. The graded Betti numbers are given by

$$
b_{i, i+1}^{\prime}=i\left(\begin{array}{c}
n+i-1 \\
i+1
\end{array}\right), \quad \text { for all } i \geq 1,
$$

and $b_{i, i+r}^{\prime}=0$, for $r>1$. To see this, note that, after a suitable linear change of variables, the ideal $I$ becomes identified with the monomial ideal $\mathfrak{m}^{2}$, where $\mathfrak{m}=\left(e_{1}, \ldots, e_{n}\right)$. Formula (2.7) then follows from general results of [1], or directly, from the exact sequence

$$
0 \longrightarrow \operatorname{Tor}_{i+1}^{E^{\prime}}(\mathbb{k}, \mathbb{k}) \longrightarrow \operatorname{Tor}_{i}^{E^{\prime}}\left(\mathfrak{m} / \mathfrak{m}^{2}(-1), \mathbb{k}\right) \longrightarrow \operatorname{Tor}_{i}^{E^{\prime}}\left(E^{\prime} / \mathfrak{m}^{2}, \mathbb{k}\right) \longrightarrow 0,
$$


where $E^{\prime}=E /\left(e_{0}\right)$, together with the isomorphism $\mathfrak{m} / \mathfrak{m}^{2} \cong \mathbb{k}^{n}$.

Going back to an arbitrary arrangement, we observe that for each flat $X \in L_{2}(\mathcal{A})$ with $\mu(X) \geq 2$ there are linear syzygies of the type discussed in the examples above, which we will call local linear syzygies. In fact, these syzygies persist in the free resolution of $A$ over $E$, as the next lemma shows.

Lemma 2.8. Let $\mathcal{A}$ be an arrangement. Then

$$
b_{i, i+1}^{\prime} \geq i \sum_{X \in L_{2}(\mathcal{A})}\left(\begin{array}{c}
\mu(X)+i-1 \\
i+1
\end{array}\right) .
$$

Moreover, if equality holds for $i=2$, then it holds for all $i \geq 2$.

Proof. Sets of local linear syzygies corresponding to different elements of $L_{2}(\mathcal{A})$ are linearly independent. Indeed, they are supported only on generators coming from the same element of $L_{2}(\mathcal{A})$.

2.9. MLS arrangements. In view of the preceding lemma, we single out a class of arrangements for which the linear strand of a minimal free resolution of $A$ over $E$ is completely determined by the Möbius function of $L_{2}(\mathcal{A})$.

Definition 2.10. An arrangement is called minimal linear strand (MLS) if

$$
b_{23}^{\prime}=2 \sum_{X \in L_{2}(\mathcal{A})}\left(\begin{array}{c}
\mu(X)+1 \\
3
\end{array}\right) .
$$

In [10], Eisenbud, Popescu, and Yuzvinsky remark that it is an interesting problem to determine explicitly a minimal free resolution of the OS-algebra over the exterior algebra. In the case of MLS arrangements in $\mathbb{C}^{3}$, Lemmas 2.4 and 2.8 permit us to compute the whole Betti diagram of the minimal resolution of $A$ over $E$, as well as the differentials in the linear strand.

Theorem 2.11. Let $\mathcal{A}$ be an $M L S$ arrangement of $n$ hyperplanes in $\mathbb{C}^{3}$. Then, the graded Betti numbers of a minimal free resolution of the OS-algebra over the exterior algebra are given by

$$
\begin{aligned}
& b_{i, i+1}^{\prime}=i \sum_{X \in L_{2}(\mathcal{A})}\left(\begin{array}{c}
\mu(X)+i-1 \\
i+1
\end{array}\right) \\
& b_{i, i+2}^{\prime}=b_{i+1, i+2}^{\prime}+\left(\begin{array}{c}
i+1 \\
2
\end{array}\right)\left(\begin{array}{c}
n+i-2 \\
i+2
\end{array}\right)-\left(\begin{array}{c}
n+i-2 \\
i
\end{array}\right) \sum_{X \in L_{2}(\mathcal{A})}\left(\begin{array}{c}
\mu(X) \\
2
\end{array}\right)
\end{aligned}
$$

Proof. The formula for $b_{i, i+1}^{\prime}$ is given by Lemma 2.8. The formula for $b_{i, i+2}^{\prime}$ follows from (2.6), which in the case $\ell=3$ boils down to

$$
1-a_{2} t^{2}+\sum_{i=1}^{\infty}(-1)^{i}\left(b_{i, i+2}^{\prime}-b_{i+1, i+2}^{\prime}\right) t^{i+2}=\frac{1+(n-1) t+\left(\left(\begin{array}{c}
n-1 \\
2
\end{array}\right)-a_{2}\right) t^{2}}{(1+t)^{n-1}} .
$$

The differentials in the linear strand are obtained in the obvious fashion from the differentials in Examples 2.6 and 2.7.

Example 2.12. Let $\mathcal{A}$ be the $X_{3}$ arrangement, with defining polynomial $Q=$ $x y z(x-z)(y+z)(y+2 x)$; see Figure 1 . We have $L_{2}^{\prime}=\left\{X_{1}, X_{2}, X_{3}\right\}$, with $\mu\left(X_{i}\right)=$ 

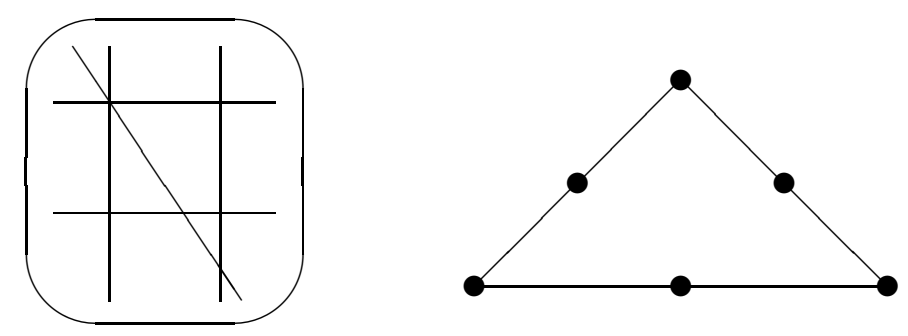

Figure 1 . The arrangement $X_{3}$ and its associated matroid

2, and so $a_{2}=3$. Moreover, $a_{3}=1$, and so condition (2.9) is satisfied. From Theorem 2.11, we compute:

$$
\begin{aligned}
& b_{i, i+1}^{\prime}=3 i, \\
& b_{i, i+2}^{\prime}=\frac{i(i+1)\left(i^{2}+5 i-2\right)}{8} .
\end{aligned}
$$

More generally, for each $n \geq 6$, consider the arrangement with defining polynomial $Q=x y z(x-z)(y+z)(y+2 x) \cdots(y+(n-4) x)$. We then have $L_{2}^{\prime}=$ $\left\{X_{1}, X_{2}, X_{3}\right\}$, with $\mu\left(X_{1}\right)=n-4, \mu\left(X_{2}\right)=\mu\left(X_{3}\right)=2$, and $a_{3}=n-5$, and so condition (2.9) is satisfied. We leave the computation of the Betti numbers $b_{i j}^{\prime}$ as an exercise.

2.13. A criterion for quadraticity. From the proof of Theorem 2.11, we see that

$$
b_{23}^{\prime}-a_{3}=b_{3}-\left(\begin{array}{c}
b_{1} \\
3
\end{array}\right)+b_{1} a_{2} .
$$

Applying Lemma 2.8, we get

$$
a_{3} \geq\left(\begin{array}{c}
b_{1} \\
3
\end{array}\right)-b_{3}-b_{1} a_{2}+2 \sum_{X \in L_{2}(\mathcal{A})}\left(\begin{array}{c}
\mu(X)+1 \\
3
\end{array}\right) .
$$

This inequality, when coupled with the definition of $a_{3}$, provides a necessary combinatorial criterion for quadraticity (and hence, Koszulness) of the OS-algebra.

Theorem 2.14. If $\left(\begin{array}{c}b_{1} \\ 3\end{array}\right)-b_{3}-b_{1} a_{2}+2 \sum_{X \in L_{2}(\mathcal{A})}\left(\begin{array}{c}\mu(X)+1 \\ 3\end{array}\right)>0$, then $A$ is not quadratic.

This criterion shows that none of the arrangements in Example 2.12 has a quadratic OS-algebra.

\section{Resolution of the Residue Field over the OS-Algebra}

We now turn to the minimal free resolution of the residue field $\mathbb{k}$ over the Orlik-Solomon algebra $A$, and to the corresponding graded Betti numbers $b_{i j}=$ $\operatorname{dim}_{\mathbb{k}} \operatorname{Tor}_{i}^{A}(\mathbb{k}, \mathbb{k})_{j}$. A 5 -term exact sequence argument relates $b_{2 j}$ to the number of minimal generators in degree $j$ of the Orlik-Solomon ideal, $a_{j}=\operatorname{dim}_{\mathbb{k}}(I[j])_{j}$. This computes the first three LCS ranks $\phi_{k}$. 
3.1. Relating various Betti numbers. Since the Hilbert series of the residue field is simply 1 , there are simple numerical constraints on the free resolution of $\mathbb{k}$ over $A$. Suppose $\mathcal{A}$ is a central, essential arrangement in $\mathbb{C}^{\ell}$. Then

$$
1=\left(1-b_{11} t+\sum_{j} b_{2 j} t^{j}-\sum_{j} b_{3 j} t^{j}+\cdots\right)\left(1+b_{1} t+b_{2} t^{2}+\cdots+b_{\ell} t^{\ell}\right) .
$$

This gives us a way to solve for $b_{r r}$, if we know all $b_{i r}$ with $i<r$ :

$$
\begin{array}{ccc}
b_{11} & = & b_{1}, \\
b_{22} & = & b_{1}^{2}-b_{2}, \\
b_{33}-b_{23} & = & b_{1}^{3}-2 b_{1} b_{2}+b_{3}, \\
b_{44}-b_{34}+b_{24} & = & b_{1}^{4}-3 b_{1}^{2} b_{2}+b_{2}^{2}+2 b_{1} b_{3}-b_{4}, \\
b_{55}-b_{45}+b_{35}-b_{25} & = & b_{1}^{5}-4 b_{1}^{3} b_{2}+3 b_{1} b_{2}^{2}+3 b_{1}^{2} b_{3}-2 b_{2} b_{3}-2 b_{1} b_{4}+b_{5},
\end{array}
$$

and, in general,

$$
\sum_{i=2}^{r}(-1)^{i} b_{i r}=\sum_{j_{1}+2 j_{2}+\cdots+r j_{r}=r} \frac{\left(j_{1}+\cdots+j_{r}\right) !}{j_{1} ! \cdots j_{r} !}\left(-b_{1}\right)^{j_{1}} \cdots\left(-b_{r}\right)^{j_{r}} .
$$

3.2. Relation with the OS-algebra. We now discuss the relation between the Betti numbers of the resolution of $\mathbb{k}$ over $A$ and the numbers $a_{j}=\operatorname{dim}_{\mathbb{k}} \operatorname{Tor}_{1}^{E}(A, \mathbb{k})_{j}$.

Lemma 3.3. We have $b_{22}=\left(\begin{array}{c}b_{1}+1 \\ 2\end{array}\right)+a_{2}$, and

$$
b_{2 j}=a_{j}, \quad \text { for } j>2 \text {. }
$$

Proof. Clearly,

$$
b_{22}=b_{1}^{2}-b_{2}=b_{1}^{2}-\left(\begin{array}{c}
b_{1} \\
2
\end{array}\right)+a_{2}=\left(\begin{array}{c}
b_{1}+1 \\
2
\end{array}\right)+a_{2} .
$$

From the change of rings spectral sequence associated to the composition of ring maps $E \rightarrow A \rightarrow \mathbb{k}$ (see \$4), we obtain a 5-term exact sequence

$$
\operatorname{Tor}_{2}^{E}(\mathbb{k}, \mathbb{k}) \longrightarrow \operatorname{Tor}_{2}^{A}(\mathbb{k}, \mathbb{k}) \longrightarrow \operatorname{Tor}_{1}^{E}(A, \mathbb{k}) \longrightarrow \operatorname{Tor}_{1}^{E}(\mathbb{k}, \mathbb{k}) \longrightarrow \operatorname{Tor}_{1}^{A}(\mathbb{k}, \mathbb{k}) \longrightarrow 0
$$

Now take free resolutions:

$$
\begin{aligned}
& \cdots \longrightarrow E^{\left(\begin{array}{c}
b_{1}+1 \\
2
\end{array}\right)}(-2) \longrightarrow E^{b_{1}}(-1) \longrightarrow \mathbb{k} \longrightarrow 0, \\
& \cdots \longrightarrow \bigoplus_{j} A^{b_{2 j}}(-j) \longrightarrow A^{b_{1}}(-1) \quad \longrightarrow \quad \longrightarrow \quad A \longrightarrow 0 \\
& \cdots \quad \longrightarrow \bigoplus_{j} E^{a_{j}}(-j) \longrightarrow E \longrightarrow 0 .
\end{aligned}
$$

The Tor's are graded, and we have the following ranks for the respective graded pieces:

\begin{tabular}{c|ccccc} 
degree & $\operatorname{Tor}_{2}^{E}(\mathbb{k}, \mathbb{k})$ & $\operatorname{Tor}_{2}^{A}(\mathbb{k}, \mathbb{k})$ & $\operatorname{Tor}_{1}^{E}(A, \mathbb{k})$ & $\operatorname{Tor}_{1}^{E}(\mathbb{k}, \mathbb{k})$ & $\operatorname{Tor}_{1}^{A}(\mathbb{k}, \mathbb{k})$ \\
\hline 0 & 0 & 0 & 0 & 0 & 0 \\
1 & 0 & 0 & 0 & $b_{1}$ & $b_{1}$ \\
2 & $\left(\begin{array}{c}b_{1}+1 \\
2\end{array}\right.$ & $b_{22}$ & $a_{2}$ & 0 & 0 \\
3 & 0 & $b_{23}$ & $a_{3}$ & 0 & 0 \\
$\vdots$ & $\vdots$ & $\vdots$ & $\vdots$ & $\vdots$ & $\vdots$ \\
$\ell$ & 0 & $b_{2 \ell}$ & $a_{\ell}$ & 0 & 0
\end{tabular}

The result follows at once. (Notice that since $b_{22}=\left(\begin{array}{c}b_{1}+1 \\ 2\end{array}\right)+a_{2}$, the sequence (3.5) is also exact on the left.) 
3.4. Witt's formula. Recall that the lower central series of a finitely-generated group $G$ is the chain of normal subgroups $G=G_{1} \geq G_{2} \geq G_{3} \geq \cdots$, with $G_{k}$ equal to the commutator $\left[G_{k-1}, G\right]$ for $k>1$ (see [23] as a basic reference). The successive quotients of the series are finitely-generated abelian groups. Let $\phi_{k}(G)=\operatorname{rank} G_{k} / G_{k+1}$ denote the rank of the $k$-th LCS quotient.

The LCS ranks of a finitely-generated free group $F_{n}$ are given by Witt's formula:

$$
\phi_{k}\left(F_{n}\right)=\frac{1}{k} \sum_{d \mid k} \mu(d) n^{\frac{k}{d}}
$$

or, equivalently,

$$
\prod_{k=1}^{\infty}\left(1-t^{k}\right)^{\phi_{k}}=1-n t
$$

For example,

$$
\phi_{1}\left(F_{n}\right)=n, \quad \phi_{2}\left(F_{n}\right)=\left(\begin{array}{l}
n \\
2
\end{array}\right), \quad \phi_{3}\left(F_{n}\right)=2\left(\begin{array}{c}
n+1 \\
3
\end{array}\right), \quad \phi_{4}\left(F_{n}\right)=\frac{n^{2}\left(n^{2}-1\right)}{4} .
$$

3.5. The first three $\phi_{k}$ 's. We now compute the ranks of the first three LCS quotients of an arrangement group, in terms of concrete combinatorial data. The results of the previous section allow us to recover a formula of Falk [11, but with a slightly new perspective. This approach illustrates that high degree syzygies which appear near the beginning of the resolution can provide information about low degree syzygies "further out". Contrast this to the approach of Peeva [27], where the Orlik-Solomon algebra is truncated in degree three.

Corollary 3.6. The ranks of the first three LCS quotients of an arrangement group are given by:

$$
\begin{aligned}
& \phi_{1}=b_{1}=\mid \text { hyperplanes } \mid \\
& \phi_{2}=\left(\begin{array}{c}
b_{1} \\
2
\end{array}\right)-b_{2}=a_{2}, \\
& \phi_{3}=b_{3}-\left(\begin{array}{c}
b_{1} \\
3
\end{array}\right)+b_{1}\left(\left(\begin{array}{c}
b_{1} \\
2
\end{array}\right)-b_{2}\right)+a_{3}=b_{3}-\left(\begin{array}{c}
b_{1} \\
3
\end{array}\right)+b_{1} a_{2}+a_{3} .
\end{aligned}
$$

Proof. As explained in 1.2 , we have

$$
\prod_{k=1}^{\infty}\left(1-t^{k}\right)^{\phi_{k}} \cdot \operatorname{Hilb}\left(\bigoplus_{i} \operatorname{Ext}_{A}^{i}(\mathbb{k}, \mathbb{k})_{i}, t\right)=1
$$

Expand both terms, using (3.2) and (3.3):

$$
\begin{gathered}
\prod_{k=1}^{\infty}\left(1-t^{k}\right)^{\phi_{k}}=1-\phi_{1} t+\left(\left(\begin{array}{c}
\phi_{1} \\
2
\end{array}\right)-\phi_{2}\right) t^{2}-\left(\left(\begin{array}{c}
\phi_{1} \\
3
\end{array}\right)-\phi_{1} \phi_{2}+\phi_{3}\right) t^{3} \\
+\left(\left(\begin{array}{c}
\phi_{1} \\
4
\end{array}\right)+\phi_{1} \phi_{3}-\left(\begin{array}{c}
\phi_{1} \\
2
\end{array}\right) \phi_{2}+\left(\begin{array}{c}
\phi_{2} \\
2
\end{array}\right)-\phi_{4}\right) t^{4}+\cdots, \\
\operatorname{Hilb}\left(\bigoplus_{i} \operatorname{Ext}_{A}^{i}(\mathbb{k}, \mathbb{k})_{i}, t\right)=1+b_{1} t+\left(b_{1}^{2}-b_{2}\right) t^{2}+\left(b_{1}^{3}-2 b_{1} b_{2}+b_{3}+a_{3}\right) t^{3}+\cdots,
\end{gathered}
$$

and solve. 
Formula (3.10) can be rewritten in terms of the Möbius function of the intersection lattice as $\phi_{2}=\sum_{X \in L_{2}(\mathcal{A})} \phi_{2}\left(F_{\mu(X)}\right)$. Formulas (3.11) and (2.12) show that $\phi_{3}$ equals the number of linear syzygies on the degree two generators of $I$ :

$$
\phi_{3}=b_{23}^{\prime} \text {. }
$$

This expression for $\phi_{3}$ (essentially the same as the one obtained by Falk in [11), depends on more subtle combinatorial data than just the Möbius function of $L(\mathcal{A})$. Nevertheless, it was shown by Falk in 12 that, for all $k \geq 3$,

$$
\phi_{k} \geq \sum_{X \in L_{2}(\mathcal{A})} \phi_{k}\left(F_{\mu(X)}\right) .
$$

For $k=3$, this inequality also follows from (2.8), together with (3.13) and (3.8).

\section{The Change of Rings spectral Sequence}

In this section, we examine the change of rings spectral sequence more deeply. This spectral sequence is due to Cartan and Eilenberg [4; a good reference for the material presented here is Eisenbud [9, appendix A.3 (see also McCleary 24], p. 508).

Our goal is to compute the Betti numbers $b_{i i}=\operatorname{dim}_{\mathbb{k}} \operatorname{Tor}_{i}^{A}(\mathbb{k}, \mathbb{k})_{i}$, which will allow us to solve for $\phi_{k}$. We concentrate on the case $k=4$, since it illustrates well the general situation. The Betti number $b_{44}$ can be obtained from $b_{34}$ and a Hilbert series computation as above, or directly from the spectral sequence. We first tackle $b_{34}$, since it corresponds to earlier terms in the spectral sequence.

4.1. Change of rings. Suppose we have a composition of ring maps:

$$
E \rightarrow A \rightarrow \mathbb{k}
$$

By taking a free resolution $Q \bullet$ for $\mathbb{k}$ over $E$ and a free resolution $P_{\bullet}$ for $\mathbb{k}$ over $A$ and tensoring with $\mathbb{k}$, we obtain a first quadrant double complex, which yields a spectral sequence

$$
{ }^{2} E^{i j} \cong \operatorname{Tor}_{i}^{A}\left(\operatorname{Tor}_{j}^{E}(A, \mathbb{k}), \mathbb{k}\right) \Longrightarrow \operatorname{Tor}_{i+j}^{E}(\mathbb{k}, \mathbb{k}) .
$$

Since ${ }_{\text {hor }}^{1} E^{i, j}=0$ unless $i=0$,

$$
{ }_{\text {hor }}^{2} E^{i, j}=\underset{\text { hor }}{\infty} E^{i, j} .
$$

In our situation (with $E \rightarrow A$ the canonical projection of the exterior algebra onto the OS-algebra, and $A \rightarrow \mathbb{k}$ the projection onto the residue field), we have

$$
{ }_{\text {hor }}^{2} E^{0, j}=\operatorname{Tor}_{j}^{E}(\mathbb{k}, \mathbb{k}),
$$

and we know how to compute this term from the Koszul complex. In particular, $\operatorname{Tor}_{j}^{E}(\mathbb{k}, \mathbb{k})$ is nonzero only in degree $j$, and we have

$$
\operatorname{dim}_{\mathbb{k}} \operatorname{Tor}_{j}^{E}(\mathbb{k}, \mathbb{k})_{j}=\left(\begin{array}{c}
b_{1}+j-1 \\
j
\end{array}\right) .
$$

We already used this fact to show that the sequence (3.5) of low degree terms was actually left exact. To keep things easy to follow, we display the $\underset{\text { vert }}{2} E^{i, j}$ terms: 


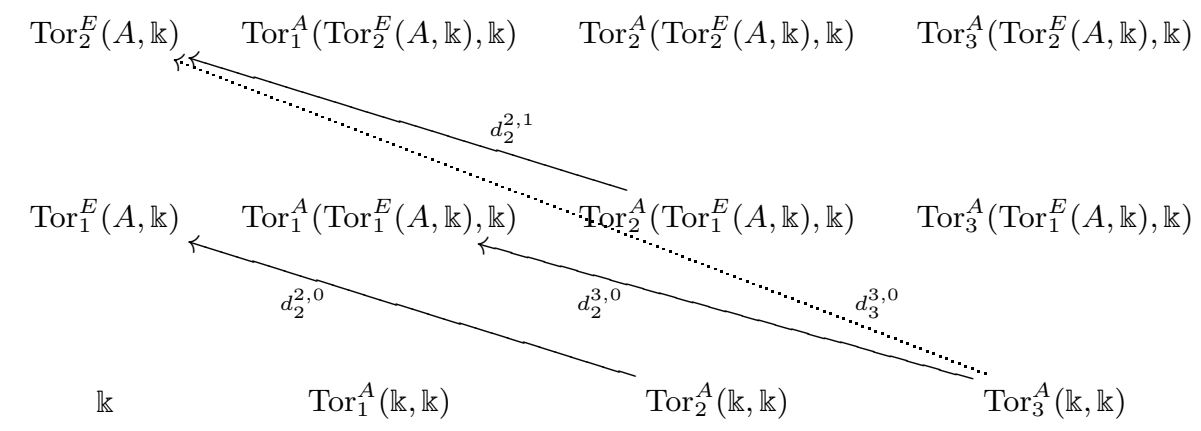

From the diagram, we compute

$$
\begin{aligned}
& \underset{\text { vert }}{\infty} E^{2,0}=\operatorname{ker} d_{2}^{2,0} \\
& \underset{\text { vert }}{\infty} E^{1,1}=\operatorname{coker} d_{2}^{3,0}, \\
& \underset{\text { vert }}{\infty} E^{0,2}=\left(\operatorname{coker} d_{2}^{2,1}\right) / d_{3}^{3,0}\left(\operatorname{ker} d_{2}^{3,0}\right) .
\end{aligned}
$$

Let tot be the total complex associated with the spectral sequence. We then have

$$
\operatorname{gr}\left(H_{k}(\text { tot })\right) \cong \bigoplus_{i+j=k} \underset{\text { vert }}{\infty} E^{i, j},
$$

and thus

$$
\operatorname{gr}\left(H_{k}(\text { tot })\right)_{r}=0, \text { for } r>k \text {. }
$$

The exactness of the sequence (3.5) of terms of low degree implies that both $\underset{\text { vert }}{\infty} E^{1,1}$ and $\underset{\text { vert }}{\infty} E^{0,2}$ vanish. Hence

$$
\begin{aligned}
\operatorname{im} d_{2}^{3,0} & =\operatorname{Tor}_{1}^{A}\left(\mathbb{k}, \operatorname{Tor}_{1}^{E}(A, \mathbb{k})\right), \\
\operatorname{coker} d_{2}^{2,1} & =d_{3}^{3,0}\left(\operatorname{ker} d_{2}^{3,0}\right) .
\end{aligned}
$$

Tracing through the spectral sequence and using the equalities above, we obtain exact sequences

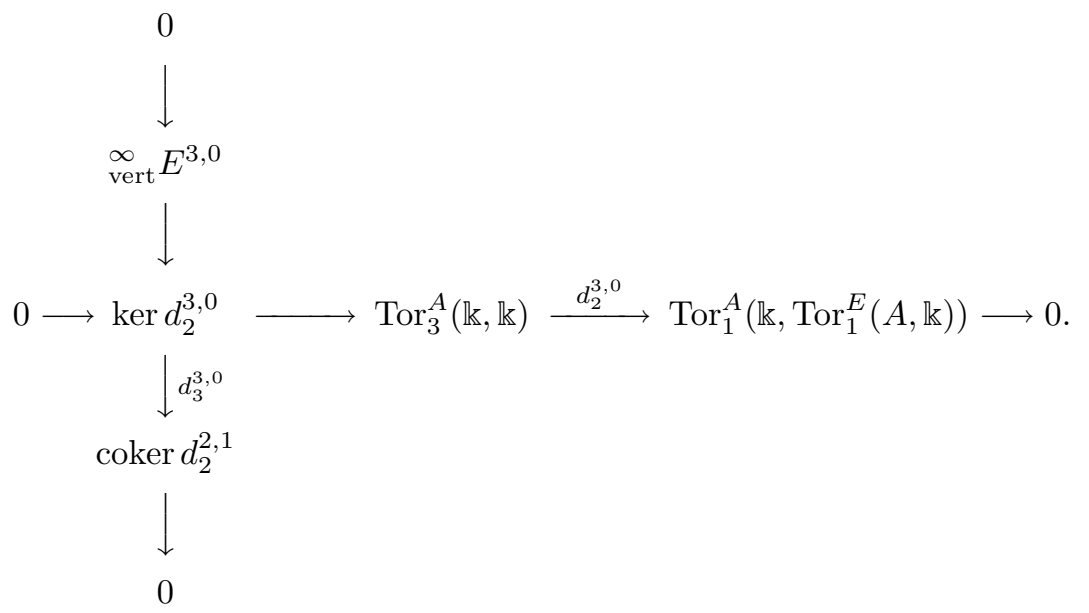


Now recall that $\operatorname{gr}\left(H_{3}\right.$ (tot) $)$ vanishes in degree greater than three, and so, in particular, $\left(\begin{array}{c}\infty \\ \text { vert }\end{array} E^{3,0}\right)_{4}=0$. We thus have proved the following theorem.

Theorem 4.2. The following sequence is exact:

$$
0 \rightarrow\left(\operatorname{coker} d_{2}^{2,1}\right)_{4} \longrightarrow \operatorname{Tor}_{3}^{A}(\mathbb{k}, \mathbb{k})_{4} \longrightarrow \operatorname{Tor}_{1}^{A}\left(\mathbb{k}, \operatorname{Tor}_{1}^{E}(A, \mathbb{k})\right)_{4} \rightarrow 0 \text {. }
$$

Since the dimension of $\operatorname{Tor}_{1}^{A}\left(\mathbb{k}, \operatorname{Tor}_{1}^{E}(A, \mathbb{k})\right)_{4}$ is $b_{1} a_{3}$, this contribution to $b_{34}=$ $\operatorname{dim}_{\mathbb{k}} \operatorname{Tor}_{3}^{A}(\mathbb{k}, \mathbb{k})_{4}$ is easy to understand. We shall analyze the contribution from $\operatorname{dim}_{\mathbb{k}}\left(\operatorname{coker} d_{2}^{2,1}\right)_{4}$ next.

4.3. Quadratic syzygies and a Koszulness test. We have already seen that if $a_{3}>0$, then $A$ is not Koszul. The sequence (4.6) gives another necessary condition for Koszulness. Indeed, if

$$
\operatorname{dim}_{\mathbb{k}} \operatorname{Tor}_{2}^{E}(A, \mathbb{k})_{4}>\operatorname{dim}_{\mathbb{k}} d_{2}^{2,1}\left(\operatorname{Tor}_{2}^{A}\left(\mathbb{k}, \operatorname{Tor}_{1}^{E}(A, \mathbb{k})\right)\right)_{4},
$$

then $A$ is not a Koszul algebra.

In order to apply this Koszulness test, we need a way to determine $\left(\operatorname{im} d_{2}^{2,1}\right)_{4}$. This is the content of the following lemma. First, recall some definitions.

Given elements $f_{1}, \ldots, f_{k} \in E$, the syzygy module is a submodule of a free module on $\epsilon_{1}, \ldots, \epsilon_{k}$, consisting of $E$-linear relations among the $f_{i}$ 's. An (exterior) Koszul syzygy is a relation of the form $f_{i} \epsilon_{j} \pm f_{j} \epsilon_{i}$. See [9] for more details.

Lemma 4.4. The image of $d_{2}^{2,1}$ in degree 4 equals $\mathcal{K}_{4}$, the space of minimal quadratic syzygies on the generators of $I_{2}$ which are Koszul.

Proof. Take (minimal) free resolutions:

$$
\begin{aligned}
& P_{\bullet}: \quad 0 \longleftarrow \mathbb{k} \longleftarrow A \longleftarrow A^{b_{1}}(-1) \longleftarrow A^{\left({ }^{b_{1}+1}\right)}(-2) \oplus A^{a_{2}}(-2) \longleftarrow \cdots, \\
& Q_{\bullet}: \quad 0 \longleftarrow \mathbb{k} \longleftarrow E \longleftarrow E^{b_{1}}(-1) \longleftarrow E^{\left({ }^{b_{1}+1}\right)}(-2) \longleftarrow \cdots,
\end{aligned}
$$

and form the double complex

$$
\begin{aligned}
& P_{0} \otimes\left(A \otimes Q_{2}\right) \underset{\leftarrow-}{\stackrel{\delta}{\kappa}} P_{1} \otimes\left(A \otimes Q_{2}\right) \quad P_{2} \otimes\left(A \otimes Q_{2}\right) \\
& P_{0} \otimes\left(A \otimes Q_{1}\right) \quad P_{1} \otimes\left(A \otimes Q_{1}\right) \stackrel{\bar{\delta}}{\longleftarrow} P_{2} \otimes\left(A \otimes Q_{1}\right) \\
& P_{0} \otimes\left(A \otimes Q_{0}\right) \quad P_{1} \otimes\left(A \otimes Q_{0}\right) \quad P_{2} \otimes\left(A \otimes Q_{0}\right)
\end{aligned}
$$

The $d_{2}$ differential is just the differential from the snake lemma. Since all the generators of $I_{2}$ may be written as products of linear forms, the result follows from a diagram chase.

In view of this lemma, we may rephrase the non-Koszul criterion (4.7), as follows.

Corollary 4.5. If the minimal resolution of $A$ over $E$ has minimal quadratic nonKoszul syzygies, then $A$ is not a Koszul algebra. 
The next lemma gives an upper bound on the number of (minimal) quadratic Koszul syzygies, solely in terms of the Möbius function of the intersection lattice of the arrangement $\mathcal{A}$.

Lemma 4.6. Let $\mathcal{K}_{4}$ be the the space of quadratic Koszul syzygies on the generators of $I_{2}$. Then

$$
\operatorname{dim}_{\mathbb{k}} \mathcal{K}_{4} \leq \sum_{(X, Y) \in\left({ }_{L_{2}(\mathcal{A})}\right)}\left(\begin{array}{c}
\mu(X) \\
2
\end{array}\right)\left(\begin{array}{c}
\mu(Y) \\
2
\end{array}\right)
$$

where $\left(\begin{array}{l}S \\ 2\end{array}\right)$ denotes the set of unordered pairs of distinct elements of a set $S$. Moreover, if $\mathcal{A}$ is $M L S$, then equality holds in (4.8).

Proof. Quadratic Koszul syzygies between elements of $I_{2}$ which come from the same dependent set are consequences of linear syzygies (because the free resolution for pencils is linear). Any two distinct elements of $L_{2}$, say $X$ and $Y$, give rise to $\left(\begin{array}{c}\mu(X) \\ 2\end{array}\right)\left(\begin{array}{c}\mu(Y) \\ 2\end{array}\right)$ quadratic Koszul syzygies, whence the bound.

In the MLS case, all linear syzygies come from pencils; hence any of the above Koszul syzygies is not a consequence of the linear syzygies.

4.7. Computing $b_{i i}$. The basic idea is to divide the problem up, using the exact sequence

$$
0 \longrightarrow \operatorname{ker} d_{2}^{i, 0} \longrightarrow \operatorname{Tor}_{i}^{A}(\mathbb{k}, \mathbb{k})_{i} \longrightarrow \operatorname{im} d_{2}^{i, 0} \longrightarrow 0
$$

We start by analyzing the kernel of $d_{2}^{i, 0}$, illustrating the method in the $i=4$ case. Using the fact that the differentials in the spectral sequence are graded, we obtain the following diagram:

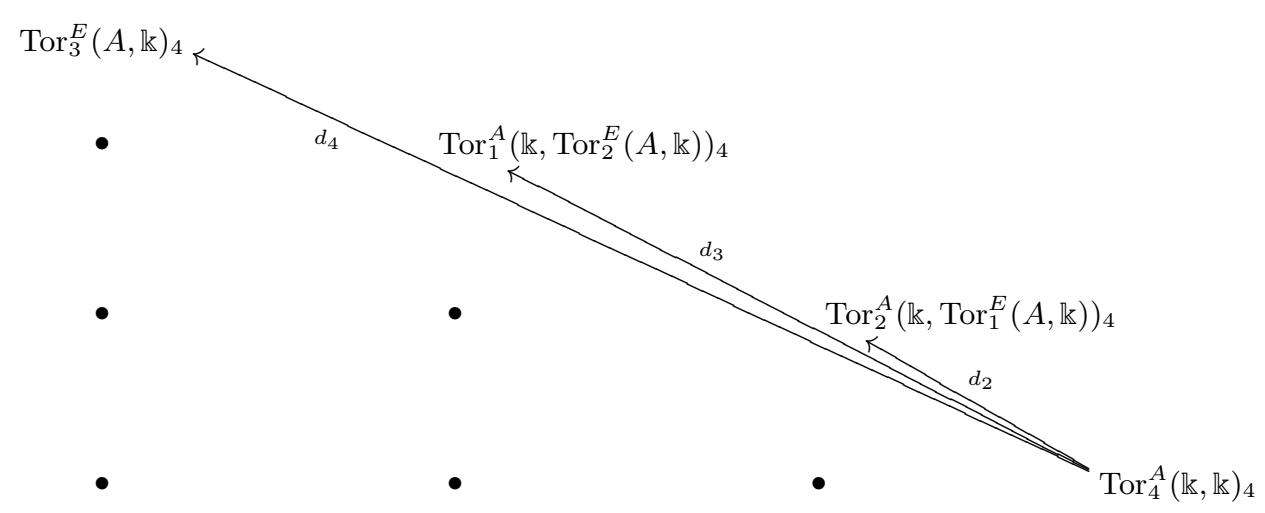

Now recall from (4.5) that $\operatorname{gr}\left(H_{4}(\text { tot })\right)_{r}=0$ if $r>4$. An analysis of the terms in $\bigoplus_{i+j=4} \underset{\text { vert }}{\infty} E^{i, j}$ shows that $\underset{\text { vert }}{\infty} E^{4,0}=\operatorname{ker} d_{4}$ is the only nonzero term, and

$$
\operatorname{dim}_{\mathbb{k}} \underset{\text { vert }}{\infty} E^{4,0}=\operatorname{dim}_{\mathbb{k}} \operatorname{Tor}_{4}^{E}(\mathbb{k}, \mathbb{k})_{4}=\left(\begin{array}{c}
b_{1}+3 \\
4
\end{array}\right) .
$$


From the diagram

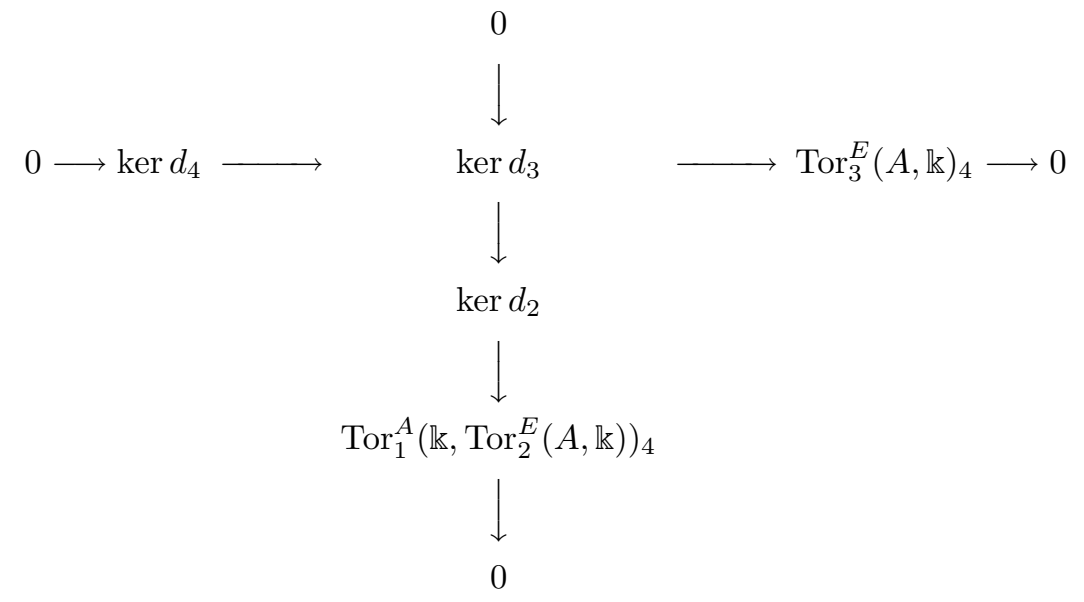

we find that

$$
\operatorname{dim}_{\mathbb{k}} \operatorname{ker} d_{2}^{4,0}=\left(\begin{array}{c}
b_{1}+3 \\
4
\end{array}\right)+b_{34}^{\prime}+b_{1} b_{23}^{\prime} .
$$

In the general case, we obtain the following.

Lemma 4.8. $\operatorname{dim}_{\mathbb{k}} \operatorname{ker} d_{2}^{i, 0}=\left(\begin{array}{c}b_{1}+i-1 \\ i\end{array}\right)+\sum_{j=0}^{i-3} b_{j j} b_{i-j-1, i-j}^{\prime}$.

Next, we analyze the image of $d_{2}^{i, 0}$. From the diagram

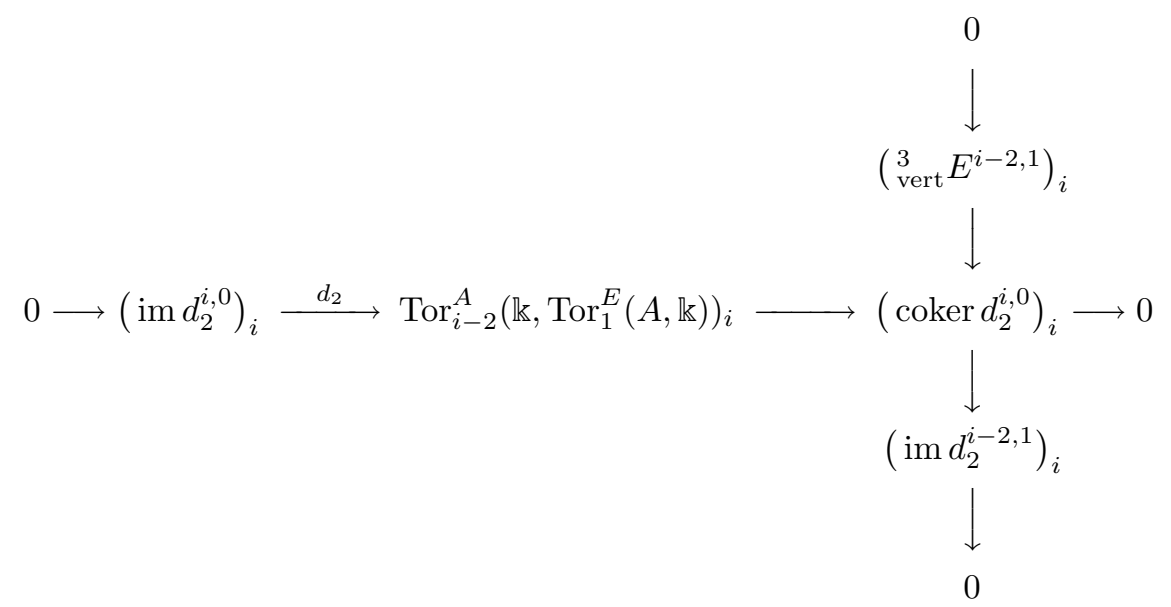

we obtain the following.

Theorem 4.9. Let $\delta_{i}=\operatorname{dim}_{\mathbb{k}} d_{2}\left(\operatorname{Tor}_{i-2}^{A}\left(\mathbb{k}, \operatorname{Tor}_{1}^{E}(A, \mathbb{k})\right)\right)_{i}+\operatorname{dim}_{\mathbb{k}}\left(\begin{array}{l}3 \\ \text { vert }\end{array} E^{i-2,1}\right)_{i}$. Then

$$
b_{i i}+\delta_{i}=\left(\begin{array}{c}
b_{1}+i-1 \\
i
\end{array}\right)+\sum_{j=0}^{i-2} b_{j j} b_{i-j-1, i-j}^{\prime} .
$$

As a consequence, we can compute the Betti numbers $b_{i i}$ inductively, provided we know all the numbers $b_{j, j+1}^{\prime}$ and $\delta_{j}$. An easy check shows that $\left(\begin{array}{l}3 \\ \text { vert }\end{array} E^{2,1}\right)_{4}=0$. 
Thus, $\delta_{4}=\operatorname{dim}_{\mathbb{k}} \operatorname{im}\left(d_{2}^{2,1}\right)_{4}$. From Lemma 4.4 we know this equals $\operatorname{dim}_{\mathbb{k}} \mathcal{K}_{4}$. We thus obtain the following.

Corollary 4.10. Let $\delta_{4}$ be the number of quadratic Koszul syzygies on the generators of $I_{2}$. Then

$$
b_{44}=\left(\begin{array}{c}
b_{1}+3 \\
4
\end{array}\right)+\left(\left(\begin{array}{c}
b_{1}+1 \\
2
\end{array}\right)+a_{2}\right) a_{2}+b_{34}^{\prime}+b_{1} b_{23}^{\prime}-\delta_{4}
$$

4.11. Computing $\phi_{4}$. From formula (1.2), and the formulas from Corollary (3.6), we find the following expression for the rank of the fourth LCS quotient:

$$
\phi_{4}=\frac{b_{1}\left(b_{1}^{3}-6 b_{1}^{2}-b_{1}-2\right)}{8}-\frac{b_{1}\left(3 b_{1}+1\right)}{2} a_{2}-\left(\begin{array}{c}
a_{2}+1 \\
2
\end{array}\right)-b_{1}\left(a_{3}+b_{3}\right)+b_{44} .
$$

Combining (4.10) and (4.11), we obtain the following.

Theorem 4.12. The rank of the fourth LCS quotient of an arrangement group $G$ is given by

$$
\phi_{4}(G)=\left(\begin{array}{c}
a_{2} \\
2
\end{array}\right)+b_{34}^{\prime}-\delta_{4} .
$$

Using the bounds for $b_{34}^{\prime}$ and $\delta_{4}$ from Lemmas 2.8 and 4.6 together with formulas (2.3) and (3.8), we recover Falk's lower bound (3.14) for $\phi_{k}$, in the particular case $k=4$.

\section{Minimal LiNEAR STRAND ARRANGEMENTS}

We now apply the machinery developed in the previous sections to the class of MLS arrangements, introduced in \$2 We start with a connection with the resonance varieties.

5.1. Local resonance. To an arbitrary arrangement $\mathcal{A}$, with Orlik-Solomon algebra $A=H^{*}(M(\mathcal{A}), \mathbb{k})$, Falk associated in 13 a sequence of resonance varieties, defined as $\mathcal{R}^{p}(\mathcal{A})=\left\{a \in E_{1} \mid H^{p}(A, \cdot a) \neq 0\right\}$. Best understood is the variety $\mathcal{R}^{1}(\mathcal{A})$, which depends only on $L_{\leq 2}(\mathcal{A})$. To each flat $X \in L_{2}(\mathcal{A})$ there corresponds a "local" component $L_{X}$ of dimension $\mu(X)$. But, in general, there are other components in $\mathcal{R}^{1}(\mathcal{A})$, as illustrated by the braid arrangements. The next result shows that non-trivial resonance cannot happen for MLS arrangements.

Theorem 5.2. If $\mathcal{A}$ is $M L S$, then all components of $\mathcal{R}^{1}(\mathcal{A})$ are local.

Proof. Suppose we have non-local resonance, i.e., there exist nonzero $a, b \in E_{1}$ with $0 \neq a \wedge b \in I_{2}$. Say, $I_{2}=\left(f_{1}, \ldots, f_{k}\right)$, so $a \wedge b=\sum c_{i} f_{i}$. Then $0=a \wedge a \wedge b=$ $a \wedge \sum c_{i} f_{i}=\sum c_{i} a \wedge f_{i}$. and Hence,

$$
\left(\begin{array}{c}
c_{1} a \\
\vdots \\
c_{k} a
\end{array}\right) \cdot\left(\begin{array}{lll}
f_{1} & \ldots & f_{k}
\end{array}\right)=0
$$

and so we have a (linear) syzygy. Since the syzygy involves $f_{1}=\left(e_{r}-e_{s}\right) \wedge\left(e_{t}-e_{s}\right)$ and, by assumption, all linear syzygies are local, the only variables that can appear in $a$ correspond to hyperplanes incident on the flat $X \in L_{2}^{\prime}(\mathcal{A})$ containing $\{r, s, t\}$. Now pick any $f_{i}$ corresponding to a flat $Y \in L_{2}^{\prime}(\mathcal{A})$ with $Y \neq X$. Since $|X \cap Y| \leq 1$, the element $a$ must be equal to some $e_{u}$, which is impossible. 
Remark 5.3. Another, more indirect proof of this theorem can be assembled from results of [5], [6]. Indeed, it can be checked that the condition (2.9) for an arrangement to be MLS coincides with the condition given in [5, Theorem 7.9] for the completion of the Alexander invariant to decompose as a direct sum of "local" Alexander invariants. From this decomposition, one infers that the variety $\mathcal{R}^{1}(\mathcal{A})$ decomposes into local components, see [6, Theorem 3.9].

Remark 5.4. The converse to Theorem 5.2 does not hold. Indeed, if $\mathcal{A}$ is a complex realization of the MacLane matroid $\mathrm{ML}_{8}$, then the Alexander invariant of $\mathcal{A}$ does not decompose (and so $\mathcal{A}$ is not MLS), even though all the components of $\mathcal{R}^{1}(\mathcal{A})$ are local, see [5, Example 8.6] and [31, Example 10.7].

5.5. LCS formula for MLS arrangements. Recall that, for any arrangement, we have $\phi_{1}=b_{1}, \phi_{2}=a_{2}$, and $\phi_{3}=b_{23}^{\prime}$. The assumption that $\mathcal{A}$ is MLS means that $\phi_{3}$ attains the lower bound (3.14) predicted by the local contributions, i.e.,

$$
\phi_{3}=2 \sum_{X \in L_{2}(\mathcal{A})}\left(\begin{array}{c}
\mu(X)+1 \\
3
\end{array}\right)=\sum_{X \in L_{2}(\mathcal{A})} \phi_{3}\left(F_{\mu(X)}\right) .
$$

The next result shows that, under this assumption, $\phi_{4}$ is given by an analogous formula.

Theorem 5.6. If $\mathcal{A}$ is an $M L S$ arrangement with group $G$, then

$$
\phi_{4}(G)=\sum_{X \in L_{2}(\mathcal{A})} \phi_{4}\left(F_{\mu(X)}\right) .
$$

Proof. By Lemmas 2.8 and 4.6 , we have

$$
\begin{aligned}
b_{i, i+1}^{\prime} & =i \sum_{X \in L_{2}(\mathcal{A})}\left(\begin{array}{c}
\mu(X)+i-1 \\
i+1
\end{array}\right), \\
\delta_{4} & =\sum_{(X, Y) \in\left(L_{2}(\mathcal{A})\right.}\left(\begin{array}{c}
\mu(X) \\
2
\end{array}\right)\left(\begin{array}{c}
\mu(Y) \\
2
\end{array}\right) .
\end{aligned}
$$

From formula (4.12), we obtain

$$
\phi_{4}=\left(\begin{array}{c}
a_{2} \\
2
\end{array}\right)+3 \sum_{X \in L_{2}(\mathcal{A})}\left(\begin{array}{c}
\mu(X)+2 \\
4
\end{array}\right)-\sum_{(X, Y) \in\left(L_{2}^{L_{2}(\mathcal{A})}\right)}\left(\begin{array}{c}
\mu(X) \\
2
\end{array}\right)\left(\begin{array}{c}
\mu(Y) \\
2
\end{array}\right) .
$$

Plugging in $a_{2}=\sum_{X \in L_{2}(\mathcal{A})}\left(\begin{array}{c}\mu(X) \\ 2\end{array}\right)$, we find that

$$
\phi_{4}=\sum_{X \in L_{2}(\mathcal{A})} \frac{\mu(X)^{2}\left(\mu(X)^{2}-1\right)}{4} .
$$

Witt's formula ends the proof.

Much of the above argument works for arbitrary $b_{i i}$ and $\phi_{k}$, though the spectral sequence chase that went into proving Corollary 4.10 becomes much more difficult. We state the expected end result as a conjecture about the LCS ranks of an MLS arrangement (the simplest case of the more general Resonance LCS Conjecture from [31]), leaving the verification for the future. 
Conjecture 5.7 (MLS LCS formula). If $G$ is the group of a minimal linear strand arrangement, then, for all $k \geq 2$,

$$
\phi_{k}(G)=\sum_{X \in L_{2}(\mathcal{A})} \phi_{k}\left(F_{\mu(X)}\right) .
$$

In other words,

$$
\prod_{k=1}^{\infty}\left(1-t^{k}\right)^{\phi_{k}}=(1-t)^{b_{1}} \prod_{X \in L_{2}(\mathcal{A})} \frac{1-\mu(X) t}{1-t} .
$$

It follows from [12, Prop. 3.12] that the conjecture holds for product 3-arrangements. Yet there are many MLS arrangements which are not products, as illustrated by the examples in $\$ 5.10$,

5.8. A simple situation. We now look at what happens in a very simple situation, namely, when $\mathcal{A}$ is MLS, and the Möbius function on $L_{2}(\mathcal{A})$ has values only 1 or 2 . This occurs precisely when $\phi_{3}=2 \phi_{2}$, or, equivalently,

$$
a_{3}+b_{3}=\left(\begin{array}{c}
b_{1} \\
3
\end{array}\right)-\left(b_{1}-2\right) a_{2} .
$$

In this situation, $b_{33}=\left(\begin{array}{c}b_{1}+2 \\ 3\end{array}\right)+\left(b_{1}+2\right) a_{2}$. Moreover,

$$
b_{i, i+1}^{\prime}=i a_{2}, \quad \text { and } \quad \delta_{4}=\left(\begin{array}{c}
a_{2} \\
2
\end{array}\right) .
$$

As a consequence, we get

Corollary 5.9. If $\phi_{3}=2 a_{2}$, then $b_{44}=\left(\begin{array}{c}b_{1}+3 \\ 4\end{array}\right)+a_{2}\left(\begin{array}{c}b_{1}+3 \\ 2\end{array}\right)+\left(\begin{array}{c}a_{2}+1 \\ 2\end{array}\right)$, and so

$$
\phi_{4}=3 a_{2} .
$$

Moreover, in this case Conjecture 5.7 takes the form $\phi_{k}(G)=a_{2} \phi_{k}\left(F_{2}\right)$, or

$$
\prod_{k=1}^{\infty}\left(1-t^{k}\right)^{\phi_{k}}=(1-t)^{b_{1}-2 a_{2}}(1-2 t)^{a_{2}} .
$$

5.10. Examples. We conclude this section with a few examples, illustrating the above formulas and conjectures.

Example 5.11 ( $X_{3}$ arrangement). The simplest example of an arrangement for which the OS-algebra is not quadratic is the $X_{3}$ arrangement from Example 2.12 We readily compute $\phi_{3}=2 a_{2}=6$; thus, the arrangement satisfies condition (5.9). In this case, formula (5.11) predicts that $\prod_{k=1}^{\infty}\left(1-t^{k}\right)^{\phi_{k}}=(1-2 t)^{3}$.

Example 5.12 (Fan arrangements). Let $\mathcal{A}$ and $\mathcal{A}^{\prime}$ be the pair of arrangements considered by Fan in [17] (see Figure 22). It is easy to see that both arrangements are MLS (in fact, they satisfy condition (5.9)).

The arrangement $\mathcal{A}$ has group $G=F_{1} \times F_{2} \times F_{2} \times F_{2}$, and thus, by Witt's formula, $\prod_{k=1}^{\infty}\left(1-t^{k}\right)^{\phi_{k}(G)}=(1-t)(1-2 t)^{3}$. The arrangement $\mathcal{A}^{\prime}$ has group $G^{\prime}=F_{1} \times G_{0}$, where $G_{0}$ is the group of the $X_{3}$ arrangement. Conjecture 5.7 predicts that $\prod_{k=1}^{\infty}\left(1-t^{k}\right)^{\phi_{k}\left(G^{\prime}\right)}=(1-t)(1-2 t)^{3}$.

In other words, the two arrangements should have the same $\phi_{k}$ 's (and thus, the same $b_{i i}$ 's), even though the respective matroids are different. Nevertheless, the combinatorial difference is picked up by the graded Betti numbers of the free 

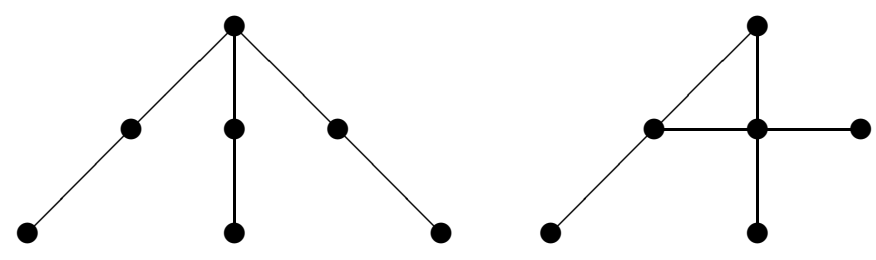

Figure 2. The Fan matroids

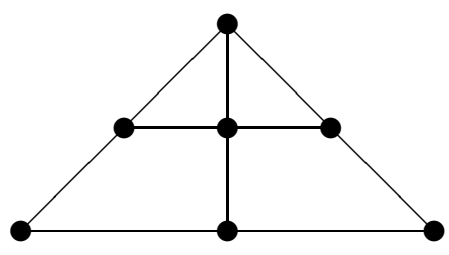

FiguRE 3. The matroid of $X_{2}$

resolutions of the respective quadratic OS-algebras. Indeed, $\operatorname{dim}_{\mathbb{k}} \operatorname{Tor}_{2}^{E}(\bar{A}, \mathbb{k})_{4}=3$, whereas $\operatorname{dim}_{\mathbb{k}} \operatorname{Tor}_{2}^{E}\left(\bar{A}^{\prime}, \mathbb{k}\right)_{4}=4$.

Example 5.13 (Kohno arrangement). An example of a quadratic OS-algebra that is not Koszul is given by Kohno's $X_{2}$ arrangement (the corresponding matroid is depicted in Figure 3). It is readily seen that $P(t)=(1+t)\left(1+6 t+10 t^{2}\right)$ and $a_{3}=0$; in particular, $A$ is quadratic. Corollary 3.6 gives $\phi_{3}=6$; hence, the arrangement satisfies condition (5.9). Conjecture 5.7 predicts that $\prod_{k=1}^{\infty}\left(1-t^{k}\right)^{\phi_{k}}=\frac{(1-2 t)^{5}}{(1-t)^{3}}$. From (5.10), we compute $b_{24}^{\prime}=15$ and $\delta_{4}=10$. Thus, $b_{34}=5$, and so the algebra $A$ is not Koszul.

Example 5.14 ( $9_{3}$ Configurations). Let $\mathcal{A}$ and $\mathcal{A}^{\prime}$ be realizations of the classical $\left(9_{3}\right)_{1}$ and $\left(9_{3}\right)_{2}$ configurations. The two arrangements have the same Möbius function, and thus, the same Poincaré polynomial: $P(t)=(1+t)\left(1+8 t+19 t^{2}\right)$. Even so, $\mathcal{A}^{\prime}$ is $\operatorname{MLS}\left(a_{3}=2\right)$, yet $\mathcal{A}$ is non-MLS $\left(a_{3}=4\right)$.

\section{Graphic arrangements}

There is a particularly nice interpretation of our results and formulas in the case of graphic arrangements.

6.1. Betti numbers and generators of the OS-ideal. Let $\mathrm{G}$ be a simple graph on $\ell$ vertices, and let $\mathcal{A}_{\mathrm{G}}$ be the corresponding graphic arrangement in $\mathbb{C}^{\ell}$, with complement $M$. The Orlik-Solomon algebra $A=H^{*}(M, \mathbb{k})$ is a quotient of the exterior algebra on generators $e_{i}$ corresponding to the edges of $\mathrm{G}$. The Poincaré polynomial is given by

$$
P(M, t)=(-t)^{\ell} \chi_{\mathrm{G}}\left(-t^{-1}\right),
$$

where $\chi_{\mathrm{G}}(t)$ is the chromatic polynomial of the graph, see [25]. A direct algorithm for computing the Betti numbers $b_{i}$ (and explicit formulas for the first four) may be 
found in Farrell [18]. As for the number of minimal generators of the Orlik-Solomon ideal, we have the following.

Lemma 6.2. For all $j>2$,

$$
a_{j}=\mid \text { chordless }(j+1) \text {-cycles } \mid .
$$

Proof. We know the $m$-cycles are dependent sets, and give rise to degree $m-1$ elements of the Orlik-Solomon ideal. The correspondence is one-to-one because a dependent set $e_{1} \cdots e_{m}$ gives a relation

$$
\prod_{i}(-1)^{i} e_{1} \cdots \hat{e}_{i} \cdots e_{m}
$$

But the lead monomial of some lower degree element of $I$ divides $e_{2} \cdots e_{m}$ iff that monomial corresponds to a chord of $\left(e_{1} \cdots e_{m}\right)$, a contradiction.

This result has been independently obtained by Cordovil and Forge [7]. Notice that the lemma does not generalize directly to arbitrary arrangements. For example, for a line configuration with four lines through a point, there are four dependent triples. But one of the OS relations can be written as a sum of the other three. This situation does not occur with graphic arrangements.

6.3. Koszulness and supersolvability. Stanley proved in 30 that a graphic arrangement $\mathcal{A}_{\mathrm{G}}$ is free iff it is supersolvable iff the graph $\mathrm{G}$ is chordal (i.e., every circuit in $\mathrm{G}$ has a chord). For a nice exposition we refer the reader to Edelman and Reiner 8 . Using Lemma 3.3 and Lemma 6.2, we obtain

Theorem 6.4. A graphic arrangement is supersolvable if and only if its OS-algebra is Koszul (in fact, quadratic).

The forward implication holds for all arrangements (cf. [29, Theorem 4.6]), but the converse is not known in general (see [32, Problem 6.7.1]). Previously, the converse was only known to hold for hypersolvable arrangements (see [20]).

6.5. The first three LCS ranks. Recall from $\$ 6.1$ that $a_{i}$ equals the number of chordless $(i+1)$-cycles in $\mathrm{G}$, for $i>2$. For each integer $r \geq 1$, define

$$
\kappa_{r-1}:=\left|\left\{\mathrm{G}^{\prime} \subseteq \mathrm{G} \mid \mathrm{G}^{\prime} \cong K_{r}\right\}\right|
$$

to be the number of complete subgraphs on $r$ vertices, so that $\kappa_{0}=\mid$ vertices $\mid=\ell$, $\kappa_{1}=\mid$ edges $\left|=b_{1}, \kappa_{2}=\right|$ triangles $\mid=a_{2}$, etc. In other words, $\left(\kappa_{0}, \kappa_{1}, \ldots, \kappa_{\ell-1}\right)$ is the $f$-vector of the "clique complex" associated to G.

Using this notation, we now answer Problem 1.4 from Falk's recent survey [14, which asks for a combinatorial formula for $\phi_{3}$ in the graphic setting.

Corollary 6.6. For a graphic arrangement, the first three LCS ranks are given by

$$
\begin{aligned}
& \phi_{1}=b_{1}=\kappa_{1} \text {, } \\
& \phi_{2}=\left(\begin{array}{c}
b_{1} \\
2
\end{array}\right)-b_{2}=\kappa_{2}, \\
& \phi_{3}=b_{3}+b_{1} a_{2}+a_{3}-\left(\begin{array}{c}
b_{1} \\
3
\end{array}\right)=2\left(\kappa_{2}+\kappa_{3}\right) .
\end{aligned}
$$

Proof. For a graphic arrangement, the first three Betti numbers are given by

$$
\begin{array}{ccc}
b_{1}= & \kappa_{1}, \\
b_{2}= & \left(\begin{array}{c}
b_{1} \\
2
\end{array}\right)-a_{2}, \\
b_{3}= & \left(\begin{array}{c}
b_{1} \\
3
\end{array}\right)-b_{1} a_{2}-a_{3}+2\left(\kappa_{2}+\kappa_{3}\right) .
\end{array}
$$

Now use Corollary 3.6. 
6.7. Resolution of a graphic OS-algebra. To proceed further, we need to analyze in more detail the resolution of the Orlik-Solomon algebra over the exterior algebra, in the case of a graphic arrangement. We start with an important example.

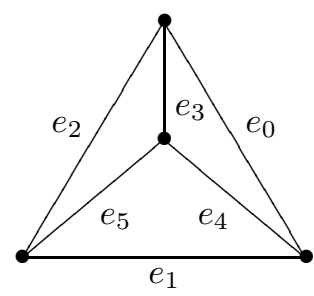

Figure 4 . The complete graph $K_{4}$

Example 6.8. The braid arrangement (in $\mathbb{C}^{4}$ ) is the graphic arrangement associated to the complete graph $K_{4}$ (see Figure 4). The free resolution of the OrlikSolomon algebra $A$ as a module over the exterior algebra $E=\Lambda^{*}\left(e_{0}, \ldots, e_{5}\right)$ begins

$$
0 \longleftarrow A \longleftarrow E \stackrel{\partial_{1}}{\longleftarrow} E^{4}(-2) \stackrel{\partial_{2}}{\longleftarrow} E^{10}(-3) \longleftarrow \cdots,
$$

where $\partial_{1}=\left(\begin{array}{llll}\partial e_{145} & \partial e_{235} & \partial e_{034} & \partial e_{012}\end{array}\right)$, and $\partial_{2}=$

$$
\left(\begin{array}{cccccccccc}
e_{1}-e_{4} & e_{1}-e_{5} & 0 & 0 & 0 & 0 & 0 & 0 & e_{3}-e_{0} & e_{2}-e_{0} \\
0 & 0 & e_{2}-e_{3} & e_{2}-e_{5} & 0 & 0 & 0 & 0 & e_{0}-e_{1} & e_{0}-e_{4} \\
0 & 0 & 0 & 0 & e_{0}-e_{3} & e_{0}-e_{4} & 0 & 0 & e_{1}-e_{5} & e_{2}-e_{5} \\
0 & 0 & 0 & 0 & 0 & 0 & e_{0}-e_{1} & e_{0}-e_{2} & e_{3}-e_{5} & e_{4}-e_{5}
\end{array}\right) .
$$

To see how this goes, write the generators of $I_{2}$ as $f_{i}=\alpha_{i} \wedge \beta_{i}(i=1, \ldots, 4)$; for example, $f_{1}=\partial e_{145}=\left(e_{1}-e_{4}\right) \wedge\left(e_{1}-e_{5}\right)$. Each $f_{i}$ generates two "local" linear syzygies, $\alpha_{i}$ and $\beta_{i}$, which appear in columns $2 i-1$ and $2 i$ of the matrix $\partial_{2}$. Let

$$
\eta_{1}=e_{0}-e_{1}-e_{3}+e_{5} \quad \text { and } \quad \eta_{2}=e_{0}-e_{2}-e_{4}+e_{5} .
$$

Note that $\eta_{1} \wedge \eta_{2}=f_{1}-f_{2}+f_{3}+f_{4} \in I_{2}$. Thus, $\eta_{1}$ and $\eta_{2}$ belong to the resonance variety $\mathcal{R}^{1}$. (In fact, $\eta_{1}$ and $\eta_{2}$ span an essential, 2-dimensional component of $\mathcal{R}^{1}$, corresponding to the neighborly partition $\Pi=(05|13| 24)$, see $[13$.$) We now get$ two new linear syzygies, expressing the fact that $\eta_{i} \wedge\left(\eta_{1} \wedge \eta_{2}\right)=0$ in $E_{3}$. Reducing modulo the "local" syzygies, we obtain the last two columns of $\partial_{2}$. It is easy to see that the columns of $\partial_{2}$ are linearly independent. From Corollary [6.6, we know that $b_{23}^{\prime}=10$. Hence, the columns of $\partial_{2}$ form a complete set of linear syzygies on $I_{2}$.

Clearly, each of these 5 pairs of linear syzygies generates $i+1$ (independent) linear $i$-th syzygies. For example, the pair $\left(\alpha_{i}, \beta_{i}\right)$ yields $\alpha_{i} \wedge \alpha_{i}=\beta_{i} \wedge \beta_{i}=$ $\alpha_{i} \wedge \beta_{i}+\beta_{i} \wedge \alpha_{i}=0$ as linear second syzygies. Hence, $b_{i, i+1}^{\prime}=5 i$, for all $i \geq 2$.

Finally, note that there are $\left(\begin{array}{l}4 \\ 2\end{array}\right)=6$ quadratic Koszul syzygies on the generators of $I_{2}$, but that they are all consequences of the linear syzygies on $I_{2}$. For example,

$$
\begin{array}{r}
\partial e_{034} \cdot \partial e_{145}-\partial e_{145} \cdot \partial e_{034}=\left(e_{0}-e_{1}\right) \cdot\left(\left(e_{3}-e_{0}\right) \partial e_{145}+\left(e_{0}-e_{1}\right) \partial e_{235}+\right. \\
\left.\left(e_{1}-e_{5}\right) \partial e_{034}+\left(e_{3}-e_{5}\right) \partial e_{012}\right)+\left(e_{0}-e_{3}\right) \cdot\left(e_{1}-e_{4}\right) \partial e_{145}+ \\
\left(e_{1}-e_{5}\right) \cdot\left(e_{0}-e_{4}\right) \partial e_{034}+\left(e_{3}-e_{5}\right) \cdot\left(e_{0}-e_{1}\right) \partial e_{012}
\end{array}
$$


It is easy to check that there are no other quadratic syzygies on the generators of $I_{2}$, and thus $b_{24}^{\prime}=0$.

Now let $\mathcal{A}=\mathcal{A}_{\mathrm{G}}$ be an arbitrary graphic arrangement.

Lemma 6.9. For $i \geq 2$,

$$
b_{i, i+1}^{\prime}=i\left(\kappa_{2}+\kappa_{3}\right) .
$$

Proof. From Corollary 6.6, we know there are $b_{23}^{\prime}=\phi_{3}=2\left(\kappa_{2}+\kappa_{3}\right)$ linear first syzygies on the generators of $I_{2}$. From the discussion in Example6.8, we know that $2 \kappa_{2}$ of those syzygies are local. Each non-local resonance component associated to a $K_{4}$ subgraph generates an additional pair of linear first syzygies, as in the example above. We claim these $2 \kappa_{3}$ linear syzygies are linearly independent.

Indeed, for there to be a linear dependence between a set of such syzygies, some pair would have to overlap at a position. Those two syzygies must involve a common element, $f$, of $I_{2}$. By assumption, the syzygies come from distinct sub- $K_{4}$ 's, so these two $K_{4}$ 's share a common triangle corresponding to $f$. Now on each triangle of a $K_{4}$, the resonant syzygies do not involve the variables of that triangle (see the above example). So the two syzygies on $f$ involving distinct $K_{4}$ 's involve different sets of variables, hence are independent. This contradiction proves the claim.

Thus, since we know that the number of linear first syzygies is $2\left(\kappa_{2}+\kappa_{3}\right)$, we have identified them all. Each pair of local linear first syzygies associated to a triangle generates $i+1$ linear $i$-th syzygies. This is also the case for the linear syzygy pairs associated to the $K_{4}$ subgraphs, as described in the example above. Such syzygies will be independent, since they are supported only on the linear syzygy pair coming from a $K_{4}$, so we are done.

Remark 6.10. In general there can be interplay between the syzygies arising from resonance components, and this will be reflected in the free resolution (this happens for example in the case of the non-Fano arrangement). But as noted above, such a phenomenon does not occur for graphic arrangements.

Lemma 6.11. $\delta_{4} \leq\left(\begin{array}{c}\kappa_{2} \\ 2\end{array}\right)-6\left(\kappa_{3}+\kappa_{4}\right)$.

Proof. There are at most $\left(\begin{array}{c}\kappa_{2} \\ 2\end{array}\right)$ quadratic Koszul syzygies. As seen in Example 6.8 the linear syzygies of a $K_{4}$-subgraph kill the 6 Koszul syzygies corresponding to that subgraph. A similar computation shows that the linear syzygies of a $K_{5}$-subgraph kill 36 Koszul syzygies. Of those, 30 are killed by the 5 sub- $K_{4}$ 's of the $K_{5}$, and the remaining 6 are killed by the $K_{5}$ itself, whence the bound.

6.12. The fourth LCS rank. We now return to the computation of the LCS ranks of an arrangement group.

Theorem 6.13. For a graphic arrangement,

$$
\phi_{4} \geq 3 \kappa_{2}+9 \kappa_{3}+6 \kappa_{4} .
$$

Furthermore, if $\kappa_{3}=0$, then $\phi_{4}=3 \kappa_{2}$.

Proof. The inequality follows from Lemmas 6.9 and 6.11, together with Theorem 4.12, If $\kappa_{3}=0$, equality follows from Corollaries 5.9 and 6.6 . 


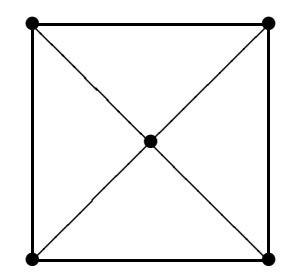

FiguRE 5. A non-hypersolvable graph

Example 6.14. Let $\mathrm{G}$ be the graph in Figure 5. The arrangement $\mathcal{A}_{\mathrm{G}}$ is the simplest example (in terms of number of vertices) of a graphic arrangement which is not hypersolvable. In particular, no general method could be applied to obtain a value for $\phi_{4}$. On the other hand, Theorem 6.13 yields $\phi_{4}=12$.

6.15. Graphic LCS formula. Extensive computations suggest that equality holds in Lemma 6.11, and hence in Theorem6.13. In fact, this seems to be part of a more general pattern, which leads us to formulate the following conjecture.

Conjecture 6.16 (Graphic LCS formula). If G is a graph with $\ell$ vertices, then

$$
\prod_{k=1}^{\infty}\left(1-t^{k}\right)^{\phi_{k}}=\prod_{j=1}^{\ell-1}(1-j t)^{\sum_{s=j}^{\ell-1}(-1)^{s-j}\left(\begin{array}{l}
s \\
j
\end{array}\right) \kappa_{s}}
$$

Expanding both sides, (6.4) becomes equivalent to the following sequence of equalities:

$$
\begin{aligned}
\phi_{1} & =\kappa_{1}, \\
\phi_{2} & =\kappa_{2}, \\
\phi_{3} & =2\left(\kappa_{2}+\kappa_{3}\right), \\
\phi_{4} & =3\left(\kappa_{2}+3 \kappa_{3}+2 \kappa_{4}\right), \\
\phi_{5}= & 6\left(\kappa_{2}+5 \kappa_{3}+8 \kappa_{4}+4 \kappa_{5}\right), \\
\phi_{6}= & 9 \kappa_{2}+89 \kappa_{3}+260 \kappa_{4}+300 \kappa_{5}+120 \kappa_{6}, \\
\vdots & \quad \vdots \\
\phi_{k}= & \sum_{j=1}^{k} \sum_{s=j}^{k}(-1)^{s-j}\left(\begin{array}{l}
s \\
j
\end{array}\right) \kappa_{s} \phi_{k}\left(F_{j}\right) .
\end{aligned}
$$

By Corollary 6.6, these equalities hold up to $k=3$. By Theorem 6.13, equality also holds for $k=4$, provided $\kappa_{3}=0$ (otherwise, we only know there is an inequality in one direction).

Remark 6.17. For graphic arrangements, the resonance formula for the ranks of the Chen groups, $\theta_{k}(G)=\operatorname{rank} \operatorname{gr}_{k}\left(G / G^{\prime \prime}\right)$ (conjectured in [31]) gives

$$
\theta_{k}=(k-1)\left(\kappa_{2}+\kappa_{3}\right), \quad \text { for } k \geq 3 .
$$


The resonance LCS formula (also conjectured in 31]) is based upon the assumption that $\phi_{4}=\theta_{4}$. In the case of graphic arrangements, formula (6.4) would imply

$$
\phi_{3}=2 \phi_{2} \Longleftrightarrow \phi_{4}=\theta_{4} \Longleftrightarrow \kappa_{3}=0
$$

Hence, if $\mathrm{G}$ contains no $K_{4}$ subgraphs, then all the above conjectures reduce to

$$
\theta_{k}=\kappa_{2} \theta_{k}\left(F_{2}\right) \text { and } \phi_{k}=\kappa_{2} \phi_{k}\left(F_{2}\right), \quad \text { for all } k \geq 2 \text {. }
$$

Remark 6.18. The graphic LCS conjecture is true for chordal graphs. Indeed, if $\mathrm{G}$ is a chordal graph, then its chromatic polynomial is given by

$$
\chi_{\mathrm{G}}(t)=t^{\kappa_{0}} \prod_{j=1}^{\kappa_{0}-1}\left(1-j t^{-1}\right)^{\sum_{s=j}^{\kappa_{0}-1}(-1)^{s-j}\left(\begin{array}{l}
s \\
j
\end{array}\right) \kappa_{s}},
$$

see 3. Since in this case $\mathcal{A}_{\mathrm{G}}$ is supersolvable, formula (6.4) follows at once from (6.1) and the Falk-Randell LCS formula (1.1). We warmly thank Yuri Volvovski for bringing formula (6.8) to our attention.

\section{REFERENCES}

[1] A. Aramova, L. Avramov, J. Herzog, Resolutions of monomial ideals and cohomology over exterior algebras, Trans. Amer. Math. Soc. 352 (2000), 579-594. MR 2000c:13021

[2] A. Beilinson, V. Ginzburg, W. Soergel, Koszul duality patterns in representation theory, J. Amer. Math. Soc. 9 (1996), 473-527. MR 96k:17010

[3] K. Braun, M. Kretz, B. Walter, M. Walter, Die chromatischen Polynome unterringfreier Graphen, Manuscripta Math. 14 (1974), 223-234. MR 50:6906

[4] H. Cartan, S. Eilenberg, Homological algebra, Princeton Univ. Press, Princeton, NJ, 1956. MR 17:1040e

[5] D. Cohen, A. Suciu, Alexander invariants of complex hyperplane arrangements, Trans. Amer. Math. Soc. 351 (1999), 4043-4067. MR 99m:52019

[6] Characteristic varieties of arrangements, Math. Proc. Cambridge Philos. Soc. 127 (1999), 33-53. MR 2000m:32036

[7] R. Cordovil, D. Forge, Quadratic Orlik-Solomon algebras of graphic matroids, http://front.math.ucdavis.edu/math.C0/0106136

[8] P. Edelman, V. Reiner, Free hyperplane arrangements between $A_{n-1}$ and $B_{n}$, Math. Z. 215 (1994), 347-365. MR 95b:52021

[9] D. Eisenbud, Commutative algebra with a view towards algebraic geometry, Graduate Texts in Math., vol. 150, Springer-Verlag, Berlin-Heidelberg-New York, 1995. MR 97a:13001

[10] D. Eisenbud, S. Popescu, S. Yuzvinsky, Hyperplane arrangement cohomology and monomials in the exterior algebra, http://front.math.ucdavis.edu/math.AG/ 9912212

[11] M. Falk, The minimal model of the complement of an arrangement of hyperplanes, Trans. Amer. Math. Soc. 309 (1988), 543-556. MR 89d:32024

[12] — The cohomology and fundamental group of a hyperplane complement, in: Singularities (Iowa City, IA, 1986), Contemporary Math., vol. 90, Amer. Math. Soc, Providence, RI, 1989, pp. 55-72. MR 90h:32026

[13] _ Arrangements and cohomology, Ann. Combin. 1 (1997), 135-157. MR 99g:52017

[14] _ Combinatorial and algebraic structure in Orlik-Solomon algebras, European J. Combinatorics 22 (2001), 687-698.

[15] M. Falk, R. Randell, The lower central series of a fiber-type arrangement, Invent. Math. 82 (1985), 77-88. MR 87c:32015b

[16] - On the homotopy theory of arrangements, II, in: Arrangements-Tokyo 1998, Adv. Stud. Pure Math., vol. 27, Math. Soc. Japan, Kinokuniya, Tokyo, 2000, pp. 93-125. MR 2002b:32044

[17] K.-M. Fan, Position of singularities and fundamental group of the complement of a union of lines, Proc. Amer. Math. Soc. 124 (1996), 3299-3303. MR 97e:14032

[18] E. J. Farrell, On chromatic coefficients, Discrete Math. 29 (1980), 257-264. MR 81d:05029 
[19] D. Grayson, M. Stillman, Macaulay 2: a software system for algebraic geometry and commutative algebra; available at http://www.math.uiuc.edu/Macaulay2

[20] M. Jambu, S. Papadima, A generalization of fiber-type arrangements and a new deformation method, Topology 37 (1998), 1135-1164. MR 99g:52019

[21] T. Kohno, Série de Poincaré-Koszul associée aux groupes de tresses pures, Invent. Math. 82 (1985), 57-75. MR 87c:32015a

[22] C. Löfwall, On the subalgebra generated by the one dimensional elements in the Yoneda Ext-algebra, in: Algebra, Algebraic Topology and Their Interactions, Lecture Notes in Math, vol. 1183, Springer-Verlag, Berlin-Heidelberg-New York, 1986, pp. 291-338. MR 88f:16030

[23] W. Magnus, A. Karrass, D. Solitar, Combinatorial group theory (2nd ed.), Dover, New York, 1976. MR 54:10423

[24] J. McCleary, A user's guide to spectral sequences, 2nd edition, Cambridge Univ. Press, Cambridge, 2001. MR 2002c:55027

[25] P. Orlik, H. Terao, Arrangements of hyperplanes, Grundlehren Math. Wiss., Bd. 300, Springer-Verlag, Berlin-Heidelberg-New York, 1992. MR 94e:52014

[26] S. Papadima, S. Yuzvinsky, On rational $K[\pi, 1]$ spaces and Koszul algebras, J. Pure Appl. Alg. 144 (1999), 156-167. MR 2000k:55017

[27] I. Peeva, Hyperplane arrangements and linear strands in resolutions, preprint, 1997.

[28] S. Priddy, Koszul resolutions, Trans. Amer. Math. Soc. 152 (1970), 39-60. MR 42:346

[29] B. Shelton, S. Yuzvinsky, Koszul algebras from graphs and hyperplane arrangements, J. London Math. Soc. 56 (1997), 477-490. MR 99c:16044

[30] R. Stanley, Supersolvable lattices, Algebra Universalis 2 (1972), 197-217. MR 46:8920

[31] A. Suciu, Fundamental groups of line arrangements: Enumerative aspects, in: Advances in algebraic geometry motivated by physics, Contemporary Math., vol. 276, Amer. Math. Soc, Providence, RI, 2001, pp. 43-79.

[32] S. Yuzvinsky, Orlik-Solomon algebras in algebra and topology, Russian Math. Surveys 56 (2001), 293-364.

Department of Mathematics, Harvard University, Cambridge, Massachusetts 02138

Current address: Department of Mathematics, Texas A\&M University, College Station, Texas 77843

E-mail address: schenck@math.tamu.edu

$U R L:$ http://www.math.tamu.edu/ schenck/

Department of Mathematics, Northeastern University, Boston, Massachusetts 02115

E-mail address: alexsuciu@neu.edu

$U R L:$ http://www.math.neu.edu/ ${ }^{\sim}$ suciu/ 